\title{
Long Range Scattering and Modified Wave Operators for the Wave-Schrödinger System III
}

\author{
J. Ginibre and G. Velo \\ Communicated by Charles Li, received April 1, 2005 and, in revised form, April 19, 2005.

\begin{abstract}
We continue the study of scattering theory for the system consisting of a Schrödinger equation and a wave equation with a Yukawa type coupling in space dimension 3. In previous papers, we proved the existence of modified wave operators for that system with no size restriction on the data and we determined the asymptotic behaviour in time of solutions in the range of the wave operators, first under a support condition on the Schrödinger asymptotic state and then without that condition, but for solutions of relatively low regularity. Here we extend the latter result to the case of more regular solutions.
\end{abstract}

\section{Contents}

1. Introduction 101

2. Notation and preliminary estimates 107

3. Cauchy problem at zero for the auxiliary system

4. Wave operators and asymptotics for (u, A) 120

$\begin{array}{ll}\text { References } & 125\end{array}$

\section{Introduction}

This paper is a sequel to two previous papers with the same title ([1] [2], hereafter referred to as I and II) where we studied the theory of scattering and proved the existence of modified wave operators for the Wave-Schrödinger (WS)

1991 Mathematics Subject Classification. Primary 35P25; Secondary 35B40, 35Q40, 81U99.

Key words and phrases. Long range scattering, modified wave operators, Wave-Schrödinger system.

Unité Mixte de Recherche (CNRS) UMR 8627.

(C)2005 International Press 
system in space dimension 3

$$
\left\{\begin{array}{l}
i \partial_{t} u=-(1 / 2) \Delta u-A u \\
\square A=|u|^{2}
\end{array}\right.
$$

where $u$ and $A$ are respectively a complex valued and a real valued function defined in space time $\mathbb{R}^{3+1}$. We refer to the introduction of I for general background and references and we give here only a general overview of the problem.

The main result of I and II was the construction of modified wave operators for the WS system, with no size restriction on the solutions. That construction basically consists in solving the Cauchy problem for the WS system with infinite initial time, namely in constructing solutions with prescribed asymptotic behaviour at infinity in time. That asymptotic behaviour is imposed in the form of suitable approximate solutions of the WS system. One then looks for exact solutions, the difference of which with the given approximate ones tends to zero at infinity in time in a suitable sense, more precisely in suitable norms. The approximate solutions are obtained as low order iterates in an iterative resolution scheme of the WS system. In I and II we used second order iterates. They are parametrized by data $\left(u_{+}, A_{+}, \dot{A}_{+}\right)$which play the role of (actually are in simpler cases) initial data at time zero. Those data constitute the asymptotic state for the actual solution.

An inherent difficulty of the WS system is the difference of propagation properties of the wave equation and of the Schrödinger equation. Because of that difficulty, we had to impose in I a support condition on the Fourier transform $F u_{+}$of the Schrödinger asymptotic state $u_{+}$, saying in effect that $F u_{+}$vanishes in a neighborhood of the unit sphere, so that $u_{+}$generates a solution of the free Schrödinger equation which is asymptotically small in a neighborhood of the light cone. Such a support condition is unpleasant because it cannot be satisfied on a dense subspace of any reasonable space where one hopes to solve the problem, typically with $u$ in $F H^{k}$ for $k>1 / 2$ ( $H^{k}$ is the standard $L^{2}$ based Sobolev space).

A progress on that problem was made by Shimomura [13] [14] who was able to construct the wave operators for the WS system without assuming that support condition, in the framework of a simpler method proposed earlier by Ozawa [11] and where the same difficulty occurs $[\mathbf{1 2}][\mathbf{1 5}]$. That method however is intrinsically restricted to the case of small Schrödinger data (see [7] for a review). The key of that progress consists in using an improved asymptotic form for the Schrödinger function, obtained by adding a term depending on $\left(A_{+}, \dot{A}_{+}\right)$which partly cancels the contribution of the asymptotic field for $A$ in the Schrödinger equation.

Although the method used in I is more complicated than the Ozawa method (so as to accomodate arbitrarily large data and solutions), it turns out that the improved asymptotic form of $u$ used in [14] can be transposed into the framework of the method of I, thereby allowing to remove the support condition on $F u_{+}$ assumed in I. This was done in II. However the treatment given in II is restricted to the case of solutions of relatively low regularity, typically with $u \in F H^{k}$ with $1<k<3 / 2$ (the case $k=1$ can also be covered by a simple variant of the same treatment). The purpose of the present paper is to extend the same result to the case of more regular solutions, namely with $k=2$. This is obtained by exploiting the fact that for the Schrödinger equation, one time derivative is homogeneous to two space derivatives, so that $H^{2}$ control of the solutions can be achieved by using only one (time) derivative. That property was extensively used in similar problems 
in $[\mathbf{4}][\mathbf{5}][\mathbf{6}][\mathbf{1 5}]$ (see [7] for a review). The methods and results of the present paper are expected to be relevant for the treatment of the corresponding problem in the general case for the more complicated Maxwell-Schrödinger system, which was considered in $[\mathbf{3}]$ in the special case of vanishing asymptotic magnetic field only.

In the remaining part of this introduction, we shall briefly review the method used in I in the modified form used in the present paper. We refer to Section 2 of I for a more detailed exposition. The main result of this paper will be stated in semi heuristic terms at the end of this introduction. The first step in that method consists in eliminating the wave equation (1.2) by solving it for $A$ and substituting the result into the Schrödinger equation, which then becomes both non linear and non local in time. One then parametrizes the Schrödinger function $u$ in terms of an amplitude $w$ and a phase $\varphi$ and one replaces the Schrödinger equation by an auxiliary system consisting of a transport equation for the amplitude and a Hamilton-Jacobi equation for the phase. One solves the Cauchy problem with prescribed asymptotic behaviour for the auxiliary system, and one finally reconstructs the solution of the original WS system from that of the auxiliary system. We now proceed to the technical details. We restrict our attention to positive time.

We first eliminate the wave equation. We define

$$
\omega=(-\Delta)^{1 / 2} \quad, \quad K(t)=\omega^{-1} \sin \omega t \quad, \quad \dot{K}(t)=\cos \omega t
$$

and we replace (1.2) by

$$
A=A_{0}+A_{1}\left(|u|^{2}\right)
$$

where

$$
\begin{gathered}
A_{0}=\dot{K}(t) A_{+}+K(t) \dot{A}_{+}, \\
A_{1}\left(|u|^{2}\right)=-\int_{t}^{\infty} d t^{\prime} K\left(t-t^{\prime}\right)\left|u\left(t^{\prime}\right)\right|^{2} .
\end{gathered}
$$

Here $A_{0}$ is a solution of the free wave equation with initial data $\left(A_{+}, \dot{A}_{+}\right)$at time $t=0$. The pair $\left(A_{+}, \dot{A}_{+}\right)$is the asymptotic state for $A$.

We next perform the change of variables mentioned above on $u$. In connection with the fact that we use time derivatives systematically in order to perform the necessary estimates, it turns out to be convenient to perform a different change of variables from that made in I and II. The main difference lies in the fact that we change $t$ into $1 / t$, so that the auxiliary system will have to be studied for $t$ in a neighborhood of zero instead of a neighborhood of infinity. The change of variables from $u$ to $w$ then becomes very similar to the pseudoconformal inversion (it would reduce to the latter if the phase $\varphi$ were absent). The unitary group

$$
U(t)=\exp (i(t / 2) \Delta)
$$

which solves the free Schrödinger equation can be written as

$$
U(t)=M(t) D(t) F M(t)
$$

where $M(t)$ is the operator of multiplication by the function

$$
M(t)=\exp \left(i x^{2} / 2 t\right),
$$

$F$ is the Fourier transform and $D(t)$ is the dilation operator

$$
D(t)=(i t)^{-3 / 2} D_{0}(t)
$$

where

$$
\left(D_{0}(t) f\right)(x)=f(x / t) .
$$


We parametrize $u$ in terms of an amplitude $w$ and of a real phase $\varphi$ as

$$
u(t)=M(t) D(t) \exp [i \varphi(1 / t)] \bar{w}(1 / t) .
$$

Substituting (1.11) into (1.1) yields an evolution equation for $(w, \varphi)$, namely

$$
\left\{i \partial_{t}+(1 / 2) \Delta-(i / 2)(2 \nabla \varphi \cdot \nabla+\Delta \varphi)+t^{-1} B+\partial_{t} \varphi-(1 / 2)|\nabla \varphi|^{2}\right\} w=0
$$

where we have expressed $A$ in terms of a new function $B$ by

$$
A(t)=t^{-1} D_{0}(t) B(1 / t) \text {. }
$$

Corresponding to the decomposition (1.3) of $A$, we decompose

$$
B=B_{0}+B_{1}(w, w)
$$

where $A_{0}(t)=t^{-1} D_{0}(t) B_{0}(1 / t)$ and $A_{1}(t)=t^{-1} D_{0}(t) B_{1}(1 / t)$. One computes easily

$$
B_{1}\left(w_{1}, w_{2}\right)=\int_{1}^{\infty} d \nu \nu^{-3} \omega^{-1} \sin ((\nu-1) \omega) D_{0}(\nu)\left(\operatorname{Re} \bar{w}_{1} w_{2}\right)(t / \nu) .
$$

At this point, we have only one evolution equation (1.12) for two functions $(w, \varphi)$. We arbitrarily impose a second equation, namely a Hamilton-Jacobi (or eikonal) equation for the phase $\varphi$, thereby splitting the equation (1.12) into a system of two equations, the other one of which being a transport type equation for the amplitude $w$. For that purpose, we split $B$ into long range and short range parts as follows. Let $\chi \in \mathcal{C}^{\infty}\left(\mathbb{R}^{3}, \mathbb{R}\right), 0 \leq \chi \leq 1, \chi(\xi)=1$ for $|\xi| \leq 1, \chi(\xi)=0$ for $|\xi| \geq 2$ and let $0<\beta<1$. We define

$$
B_{0}=B_{0 L}+B_{0 S} \quad, \quad B_{1}=B_{L}+B_{S}
$$

where

$$
\left\{\begin{array}{l}
F B_{0 L}(t, \xi)=\chi\left(\xi t^{\beta}\right) F B_{0}(t, \xi), \\
F B_{L}(t, \xi)=\chi\left(\xi t^{\beta}\right) F B_{1}(t, \xi) .
\end{array}\right.
$$

The parameter $\beta$ will have to satisfy various conditions which will appear later, all of them compatible with $\beta=1 / 3$.

We split the equation (1.12) into the following system of two equations.

$$
\left\{\begin{array}{l}
i \partial_{t} w+(1 / 2) \Delta w=i Q(\nabla \varphi, w)-t^{-1}\left(B_{0 S}+B_{S}(w, w)\right) w \\
\partial_{t} \varphi=(1 / 2)|\nabla \varphi|^{2}-t^{-1} B_{0 L}-t^{-1} B_{L}(w, w)
\end{array}\right.
$$

where we have defined

$$
Q(s, w)=s \cdot \nabla w+(1 / 2)(\nabla \cdot s) w
$$

for any vector field $s$. The first equation of (1.18) is the transport type equation for the amplitude $w$, while the second one is the Hamilton-Jacobi type equation for the phase $\varphi$. Since the right-hand sides of (1.18) contain $\varphi$ only through its gradient, we can obtain from (1.18) a closed system for $w$ and $s=\nabla \varphi$ by taking the gradient of the second equation, namely

$$
\left\{\begin{array}{l}
i \partial_{t} w+(1 / 2) \Delta w=i Q(s, w)-t^{-1}\left(B_{0 S}+B_{S}(w, w)\right) w \\
\partial_{t} s=s \cdot \nabla s-t^{-1} \nabla B_{0 L}-t^{-1} \nabla B_{L}(w, w) .
\end{array}\right.
$$


Once the system $(1.20)$ is solved for $(w, s)$, one recovers $\varphi$ easily by integrating the second equation of (1.18) over time. The system (1.20) will be referred to as the auxiliary system.

The construction of the modified wave operators follows the same pattern as in I and II. The first task is to construct solutions of the auxiliary system (1.20) with suitably prescribed asymptotic behaviour at zero, and in particular with $w(t)$ tending to a limit $w_{+}=F u_{+}$as $t \rightarrow 0$. That asymptotic behaviour is imposed in the form of a suitably chosen pair $(W, \phi)$ and therefore $(W, S)$ with $S=\nabla \phi$ with $W(t)$ tending to $w_{+}$as $t \rightarrow 0$. For fixed $(W, S)$, we make a change of variables in the system $(1.18)$ from $(w, \varphi)$ to $(q, \psi)$ defined by

$$
(q, \psi)=(w, \varphi)-(W, \phi)
$$

or equivalently a change of variables in the system $(1.20)$ from $(w, s)$ to $(q, \sigma)$ defined by

$$
(q, \sigma)=(w, s)-(W, S)
$$

and instead of looking for a solution $(w, s)$ of the system $(1.20)$ with $(w, s)$ behaving asymptotically as $(W, S)$, we look for a solution $(q, \sigma)$ of the transformed system with $(q, \sigma)$ (and also $\psi$ ) tending to zero as $t \rightarrow 0$. Performing the change of variables (1.22) in the auxiliary system (1.20) yields the following modified auxiliary system for the new variables $(q, \sigma)$

$$
\left\{\begin{array}{l}
i \partial_{t} q+(1 / 2) \Delta q=i(Q(s, q)+Q(\sigma, W))-t^{-1} B_{0 S} q \\
-t^{-1} B_{S}(w, w) q-t^{-1}\left(2 B_{S}(W, q)+B_{S}(q, q)\right) W-R_{1}(W, S) \\
\partial_{t} \sigma=(s \cdot \nabla \sigma+\sigma \cdot \nabla S)-t^{-1} \nabla\left(2 B_{L}(W, q)+B_{L}(q, q)\right)-R_{2}(W, S),
\end{array}\right.
$$

where the remainders $R_{1}(W, S)$ and $R_{2}(W, S)$ are defined by

$$
\begin{gathered}
R_{1}(W, S)=i \partial_{t} W+(1 / 2) \Delta W-i Q(S, W)+t^{-1}\left(B_{0 S}+B_{S}(W, W)\right) W \\
R_{2}(W, S)=\partial_{t} S-S \cdot \nabla S+t^{-1} \nabla B_{0 L}+t^{-1} \nabla B_{L}(W, W)
\end{gathered}
$$

and the dependence of the remainders on $B_{0}$ has been omitted in the notation. For technical reasons, it is useful to consider also a partly linearized version of the system (1.23), namely

$$
\left\{\begin{array}{l}
i \partial_{t} q^{\prime}+(1 / 2) \Delta q^{\prime}=i\left(Q\left(s, q^{\prime}\right)+Q(\sigma, W)\right)-t^{-1} B_{0 S} q^{\prime} \\
-t^{-1} B_{S}(w, w) q^{\prime}-t^{-1}\left(2 B_{S}(W, q)+B_{S}(q, q)\right) W-R_{1}(W, S) \\
\partial_{t} \sigma^{\prime}=\left(s \cdot \nabla \sigma^{\prime}+\sigma \cdot \nabla S\right)-t^{-1} \nabla\left(2 B_{L}(W, q)+B_{L}(q, q)\right)-R_{2}(W, S) .
\end{array}\right.
$$

The construction of solutions $(w, s)$ of the auxiliary system (1.20) defined for small time and with prescribed behaviour $(W, S)$ at zero proceeds in two steps. The first step consists in solving the system $(1.23)$ for $(q, \sigma)$ tending to zero at zero under suitable boundedness properties of $B_{0}$ and $(W, S)$ and suitable vanishing properties of the remainders $R_{1}(W, S)$ and $R_{2}(W, S)$ at zero, by a minor variation of the method used in I. That method consists in first solving the linearized system $(1.26)$ for $\left(q^{\prime}, \sigma^{\prime}\right)$ with given $(q, \sigma)$, and then showing that the map $(q, \sigma) \rightarrow\left(q^{\prime}, \sigma^{\prime}\right)$ thereby defined has a fixed point, by the use of a contraction method. The second step consists in constructing $(W, S)$ with $W(t)$ tending to $w_{+}$as $t \rightarrow 0$ and satisfying the required boundedness and vanishing properties. This is done by solving the 
auxiliary system (1.20) by iteration to second order as in I and then adding to $W$ an additional term of the same form as that used in [14] and in II. The detailed form of $(W, S)$ thereby obtained is too complicated to be given here and will be given in Section 3 below (see (3.42)-(3.47)).

Once the system $(1.20)$ is solved for $(w, s)$, one can proceed therefrom to the construction of a solution $(u, A)$ of the original WS system. One first defines the phases $\varphi$ and $\phi$ such that $s=\nabla \varphi$ and $S=\nabla \phi$ and one reconstructs $(u, A)$ from $(w, \varphi)$ by $(1.11)(1.3)(1.5)$, thereby obtaining a solution of the WS system defined for large time and with prescribed asymptotic behaviour. The modified wave operator for the WS system is then defined as the map $\Omega:\left(u_{+}, A_{+}, \dot{A}_{+}\right) \rightarrow(u, A)$.

The main result of this paper is the construction of $(u, A)$ from $\left(u_{+}, A_{+}, \dot{A}_{+}\right)$as described above, together with the asymptotic properties of $(u, A)$ that follow from that construction. It will be stated below in full mathematical detail in Proposition 4.1. We give here a heuristic preview of that result, stripped from most technicalities. We set $\beta=1 / 3$ for definiteness.

Proposition 1.1. Let $\beta=1 / 3$. Let $\left(u_{+}, A_{+}, \dot{A}_{+}\right)$be such that $w_{+}=F u_{+} \in H^{k_{+}}$ for sufficiently large $k_{+}$, that $\left(A_{+}, \dot{A}_{+}\right)$be sufficiently regular, and that $\left(F A_{+}, F \dot{A}_{+}\right)$ be sufficiently small near $\xi=0$. Let $(W, S)$ be the approximate solution of the system (1.20) defined by (3.42)-(3.47). Then

(1) There exists $\tau=\tau\left(u_{+}, A_{+}, \dot{A}_{+}\right), 0<\tau \leq 1$, such that the auxiliary system (1.20) has a unique solution $(w, s)$ in a suitable space, defined for $0<t \leq \tau$ and such that $(w-W, s-S)$ tends to zero in suitable norms when $t \rightarrow 0$.

(2) There exists $\varphi$ and $\phi$ such that $s=\nabla \varphi, S=\nabla \phi, \phi(1)=0$ and such that $\varphi-\phi$ tends to zero in suitable norms when $t \rightarrow 0$. Define $(u, A)$ by (1.11) (1.3) (1.5). Then $(u, A)$ solves the system (1.1) (1.2) for $t \geq T=\tau^{-1}$ and $(u(t), A(t))$ behaves asymptotically as $\left(M(t) D(t) \exp (i \phi(1 / t)) \bar{W}(1 / t), A_{0}+A_{1}\left(|D(t) W(1 / t)|^{2}\right)\right)$ in the sense that the difference tends to zero in suitable norms (for which each term separately is $O(1))$ when $t \rightarrow \infty$.

The unspecified condition that $\left(F A_{+}, F \dot{A}_{+}\right)$be sufficiently small near $\xi=0$ can be shown to follow from more intuitive conditions in $x$-space, consisting of decay conditions at infinity in space, and, depending on the values of the parameters defining the relevant function spaces, of some moment conditions on $\left(A_{+}, \dot{A}_{+}\right)$.

This paper relies on a large amount of material from I and II. In order to bring out the structure while keeping duplication to a minimum, we give without proof a shortened logically self-sufficient sequence of those intermediate results from I and II that are needed, and we provide a full exposition only for the parts that are new as compared with I and II. When quoting I or II, we shall use the notation (I.p.q) or (II.p.q) for equation (p.q) of I or II and Item I.p.q or II.p.q for Item p.q of I or II, such as Lemma, Proposition, etc.

The remaining part of this paper is organized as follows. In Section 2 we collect notation and some estimates of a general nature. In Section 3, we study the Cauchy problem at zero for the auxiliary system (1.20). We first prove the existence of solutions under suitable boundedness properties of $(W, S)$ and suitable vanishing properties of the remainders at zero (Proposition 3.1). We then define $(W, S)$ and prove that they satisfy the previous properties, which yields the main result on the Cauchy problem at zero for the auxiliary system (1.20) (Proposition 
3.2). Finally in Section 4, we construct the wave operators for the WS system (1.1) (1.2) and we derive the asymptotic properties of the solution $(u, A)$ in their range that follow from the previous results (Proposition 4.1).

\section{Notation and preliminary estimates}

In this section we introduce some notation and we collect a number of estimates which will be used throughout this paper. We denote by $\|\cdot\|_{r}$ the norm in $L^{r} \equiv L^{r}\left(\mathbb{R}^{3}\right)$ and we define $\delta(r)=3 / 2-3 / r$. For any interval $I$ and any Banach space $X$ we denote by $\mathcal{C}(I, X)$ the space of strongly continuous functions from $I$ to $X$ and by $L^{\infty}(I, X)$ the space of measurable essentially bounded functions from $I$ to $X$. For real numbers $a$ and $b$ we use the notation $a \vee b=\operatorname{Max}(a, b)$ and $a \wedge b=\operatorname{Min}(a, b)$. In the estimates of solutions of the relevant equations we shall use the letter $\mathrm{C}$ to denote constants, possibly different from an estimate to the next, depending on various parameters but in general not on the solutions themselves or on their initial data. We shall use the notation $C\left(a_{1}, a_{2}, \cdots\right)$ for estimating functions, also possibly different from an estimate to the next, depending on suitable norms $a_{1}, a_{2}, \cdots$ of the solutions or of their initial data.

We shall use the Sobolev spaces $\dot{H}_{r}^{k}$ and $H_{r}^{k}$ defined for $-\infty<k<+\infty$, $1 \leq r \leq \infty$ by

$$
\dot{H}_{r}^{k}=\left\{u:\left\|u ; \dot{H}_{r}^{k}\right\| \equiv\left\|\omega^{k} u\right\|_{r}<\infty\right\}
$$

and

$$
H_{r}^{k}=\left\{u:\left\|u ; H_{r}^{k}\right\| \equiv\left\|<\omega>^{k} u\right\|_{r}<\infty\right\}
$$

where $\omega=(-\Delta)^{1 / 2}$ and $\langle\cdot\rangle=\left(1+|\cdot|^{2}\right)^{1 / 2}$. The subscript $r$ will be omitted if $r=2$ and we shall use the notation $\left\|w ; H^{k}\right\|=|w|_{k}$.

We shall look for solutions of the auxiliary system (1.20) such that $(w, \nabla s) \in$ $\mathcal{C}\left(I, H^{k} \oplus H^{\ell}\right)$ where $I$ is an interval and where it is understood that $\nabla s \in L^{2}$ includes the fact that $s \in L^{6}$.

We shall use extensively the following Sobolev inequalities, stated here in $\mathbb{R}^{n}$, but to be used only for $n=3$.

Lemma 2.1. Let $1<q, r<\infty, 1<p \leq \infty$ and $0 \leq j<k$. If $p=\infty$, assume that $k-j>n / r$. Let $\sigma$ satisfy $j / k \leq \sigma \leq 1$ and

$$
n / p-j=(1-\sigma) n / q+\sigma(n / r-k) .
$$

Then the following inequality holds

$$
\left\|\omega^{j} u\right\|_{p} \leq C\|u\|_{q}^{1-\sigma}\left\|\omega^{k} u\right\|_{r}^{\sigma} .
$$

The proof follows from the Hardy-Littlewood-Sobolev (HLS) inequality ([8], p. 117) (from the Young inequality if $p=\infty$ ), from Paley-Littlewood theory and interpolation.

We shall also use extensively the following Leibnitz and commutator estimates.

Lemma 2.2. Let $1<r, r_{1}, r_{3}<\infty$ and

$$
1 / r=1 / r_{1}+1 / r_{2}=1 / r_{3}+1 / r_{4} .
$$


Then the following estimates hold

$$
\left\|\omega^{m}(u v)\right\|_{r} \leq C\left(\left\|\omega^{m} u\right\|_{r_{1}}\|v\|_{r_{2}}+\left\|\omega^{m} v\right\|_{r_{3}}\|u\|_{r_{4}}\right)
$$

for $m \geq 0$, and

$$
\left\|\left[\omega^{m}, u\right] v\right\|_{r} \leq C\left(\left\|\omega^{m} u\right\|_{r_{1}}\|v\|_{r_{2}}+\left\|\omega^{m-1} v\right\|_{r_{3}}\|\nabla u\|_{r_{4}}\right)
$$

for $m \geq 1$, where $[$,$] denotes the commutator.$

The proof of those estimates is given in [9] [10] with $\omega$ replaced by $\langle\omega\rangle$ and follows therefrom by a scaling argument.

We next give some estimates of $B_{0 L}, B_{0 S}, B_{L}$ and $B_{S}$ defined by (1.16) (1.17). It follows immediately from (1.16) (1.17) that

$$
\left\|\omega^{m} B_{0 L}\right\|_{2} \leq\left(2 t^{-\beta}\right)^{m-p}\left\|\omega^{p} B_{0 L}\right\|_{2} \leq\left(2 t^{-\beta}\right)^{m-p}\left\|\omega^{p} B_{0}\right\|_{2}
$$

for $m \geq p$ and

$$
\left\|\omega^{m} B_{0 S}\right\|_{2} \leq t^{\beta(p-m)}\left\|\omega^{p} B_{0 S}\right\|_{2} \leq t^{\beta(p-m)}\left\|\omega^{p} B_{0}\right\|_{2}
$$

for $m \leq p$. Similar estimates hold for $B_{L}, B_{S}$. We shall need estimates of $B_{1}$ defined by (1.15) and of $\partial_{t} B_{1}$. From (1.15) it follows that

$$
\partial_{t} B_{1}\left(w_{1}, w_{2}\right)=\widetilde{B}_{1}\left(\partial_{t} w_{1}, w_{2}\right)+\widetilde{B}_{1}\left(w_{1}, \partial_{t} w_{2}\right)
$$

where

$$
\widetilde{B}_{1}\left(w_{1}, w_{2}\right)=\int_{1}^{\infty} d \nu \nu^{-4} \omega^{-1} \sin ((\nu-1) \omega) D_{0}(\nu)\left(\operatorname{Re}\left(\bar{w}_{1} w_{2}\right)\right)(t / \nu) .
$$

It follows from (1.15) (2.7) that

$$
\left\{\begin{array}{l}
\left\|\omega^{m+1} B_{1}\left(w_{1}, w_{2}\right)\right\|_{2} \leq I_{m}\left(\left\|\omega^{m}\left(\bar{w}_{1} w_{2}\right)\right\|_{2}\right) \\
\left\|\omega^{m+1} \widetilde{B}_{1}\left(w_{1}, w_{2}\right)\right\|_{2} \leq I_{m+1}\left(\left\|\omega^{m}\left(\bar{w}_{1} w_{2}\right)\right\|_{2}\right)
\end{array}\right.
$$

where $I_{m}$ is defined by

$$
\left(I_{m}(f)\right)(t)=\int_{1}^{\infty} d \nu \nu^{-m-3 / 2} f(t / \nu) .
$$

We finally collect some estimates of the solutions of the free wave equation $\square A_{0}=0$ with initial data $\left(A_{+}, \dot{A}_{+}\right)$at time zero, given by (1.4).

Lemma 2.3. Let $k \geq 0$. Let $A_{+}$and $\dot{A}_{+}$satisfy the conditions

$$
A_{+}, \omega^{-1} \dot{A}_{+} \in H^{k} \quad, \quad \nabla^{2} A_{+}, \nabla \dot{A}_{+} \in H_{1}^{k} .
$$

Then the following estimate holds :

$$
\left\|\omega^{m} A_{0}\right\|_{r} \leq b_{0} t^{-1+2 / r} \quad \text { for } 2 \leq r \leq \infty,
$$

for $0 \leq m \leq k$ and for all $t>0$, where $b_{0}$ depends on $\left(A_{+}, \dot{A}_{+}\right)$through the norms associated with (2.10).

The estimate (2.11) can be expressed in an equivalent form in terms of $B_{0}$ defined by (1.13), namely

$$
\left\|\omega^{m} B_{0}\right\|_{r} \leq b_{0} t^{-m+1 / r} \quad \text { for } 2 \leq r \leq \infty .
$$


We shall also need some estimates on time derivatives of $B_{0}$. From the fact that the dilation generator $P=t \partial_{t}+x \cdot \nabla$ satisfies the commutation relation

$$
P=D_{0}(t) t \partial_{t} D_{0}(t)^{-1},
$$

and from (1.13), it follows that for any non negative integer $j$

$$
(P+1)^{j} A_{0}(t)=(-)^{j} t^{-1} D_{0}(t)\left(\left(t \partial_{t}\right)^{j} B_{0}\right)(1 / t) .
$$

On the other hand if $A_{0}$ is a solution of the wave equation $\square A_{0}=0$ with initial data $\left(A_{+}, \dot{A}_{+}\right)$at $t=0$, then also $P A_{0}$ is a solution of the same equation, with initial data $x \cdot \nabla A_{+}$and $(1+x \cdot \nabla) \dot{A}_{+}$. Combining the previous remarks with Lemma 2.3, we obtain the following corollary.

Corollary 2.1. Let $A_{+}$and $\dot{A}_{+}$satisfy the conditions

$$
\begin{aligned}
A_{+}, \omega^{-1} \dot{A}_{+} \in L^{2} & , \quad x \cdot \nabla A_{+}, \omega^{-1} x \cdot \nabla \dot{A}_{+} \in L^{2} . \\
\nabla^{2} A_{+}, \nabla \dot{A}_{+} \in L^{1} & , \quad \nabla^{2} x \cdot \nabla A_{+}, \nabla x \cdot \nabla \dot{A}_{+} \in L^{1} .
\end{aligned}
$$

Then $B_{0}$ defined by (1.13) satisfies the estimates

$$
\left\|\partial_{t}^{j} B_{0}\right\|_{r} \leq b_{0} t^{-j+1 / r}
$$

for $j=0,1$ and for $2 \leq r \leq \infty$, where $b_{0}$ depends on $\left(A_{+}, \dot{A}_{+}\right)$through the norms associated with (2.15) (2.16).

Finally from the fact that $\chi \in \mathcal{C}_{0}^{\infty}$ and from obvious scaling properties, it follows that $B_{0 S}$ and $B_{0 L}$ also satisfy the estimates (2.12) (2.17), possibly up to an absolute constant.

\section{Cauchy problem at zero for the auxiliary system}

In this section, we solve the Cauchy problem with initial time zero for the auxiliary system (1.20) in the difference form (1.23). We first solve the system (1.23) for $(q, \sigma)$ tending to zero at zero under suitable boundedness properties of $\left(B_{0}, W, S\right)$ and suitable vanishing properties of the remainders $R_{1}(W, S)$ and $R_{2}(W, S)$. We then construct $(W, S)$ with $W(t)$ tending to $w_{+}=F u_{+}$as $t \rightarrow 0$ and satisfying the required boundedness and vanishing properties. The method closely follows that of Sections 6 and 7 of I.

We first estimate a single solution of the linearized auxiliary system (1.26) at the level of regularity where we shall eventually solve the auxiliary system (1.20). The following lemma is a variant of Lemma I.6.1 with $k=2$, where however the second order space derivatives of $q$ are estimated through the use of the time derivative.

Lemma 3.1. Let $\beta>0$ and $\ell>3 / 2$. Let $\tau \leq 1$ and $I=(0, \tau]$. Let $B_{0} \in \mathcal{C}^{1}\left(I, L^{\infty}\right)$ satisfy the estimate (2.17) for $r=\infty$ and $j=0,1$. Let $(W, \nabla S) \in \mathcal{C}\left(I, H^{2} \oplus\right.$ $\left.H^{\ell+1}\right) \cap \mathcal{C}^{1}\left(I, H^{1} \oplus H^{\ell-1}\right)$ and let $\left(B_{0}, W, S\right)$ be such that $R_{1} \in \mathcal{C}^{1}\left(I, L^{2}\right)$ and $\nabla R_{2} \in \mathcal{C}\left(I, H^{\ell}\right)$. Let $(q, \nabla \sigma),\left(q^{\prime}, \nabla \sigma^{\prime}\right) \in \mathcal{C}\left(I, H^{2} \oplus H^{\ell}\right) \cap \mathcal{C}^{1}\left(I, L^{2} \oplus H^{\ell-1}\right)$ and let $\left(q^{\prime}, \sigma^{\prime}\right)$ be a solution of the system (1.26) in I. Assume that $W$ and $q$ satisfy

$$
\operatorname{Sup}_{t \in I}\left(\|W\|_{\infty} \vee|W|_{3 / 2} \vee\|q\|_{\infty} \vee|q|_{3 / 2}\right) \leq a
$$


for all $t \in I$. Then the following estimates hold for all $t \in I$ :

$$
\begin{aligned}
& \left|\partial_{t}\left\|q^{\prime}\right\|_{2}\right| \leq C\left\{a\|\nabla \sigma\|_{2}+t^{-1+\beta} a^{2} I_{0}\left(\|q\|_{2}\right)\right\}+\left\|R_{1}(W, S)\right\|_{2} \\
& \left\|\Delta q^{\prime}\right\|_{2} \leq C\left\{\left\|\partial_{t} q^{\prime}\right\|_{2}+\left(\|s\|_{\infty}+\|\nabla \cdot s\|_{3}\right)^{2}\left\|q^{\prime}\right\|_{2}+a\|\nabla \sigma\|_{2}\right. \\
& \left.+t^{-1}\left(b_{0}+a^{2} t^{\beta}\right)\left\|q^{\prime}\right\|_{2}+t^{-1+\beta} a^{2} I_{0}\left(\|q\|_{2}\right)+\left\|R_{1}(W, S)\right\|_{2}\right\}
\end{aligned}
$$

where $s=S+\sigma$,

$$
\begin{aligned}
& \left|\partial_{t}\left\|\partial_{t} q^{\prime}\right\|_{2}\right| \leq C\left\{\left(\left\|\partial_{t} s\right\|_{\infty}+\| \partial_{t} \nabla \cdot s\right) \|_{3}\right)\left\|\nabla q^{\prime}\right\|_{2}+a\left\|\partial_{t} \nabla \sigma\right\|_{2} \\
& +\left(\|\sigma\|_{\infty}+\|\nabla \cdot \sigma\|_{3}\right)\left\|\nabla \partial_{t} W\right\|_{2}+t^{-2}\left(b_{0}+a^{2} t^{\beta}\right)\left\|q^{\prime}\right\|_{2} \\
& +t^{-2+\beta} a^{2} I_{0}\left(\|q\|_{2}\right)+t^{-1} a\left(\left\|\partial_{t} W\right\|_{3} I_{0}\left(\|q\|_{2}\right)+I_{0}\left(\left\|\partial_{t} W\right\|_{3}\|q\|_{2}\right)\right) \\
& \left.\left.+t^{-1+\beta} a^{2} I_{1}\left(\left\|\partial_{t} q\right\|_{2}\right)+t^{-1} a I_{1}\left(\| \partial_{t} W\right)\left\|_{2}+\right\| \partial_{t} q \|_{2}\right)\left\|q^{\prime}\right\|_{3}\right\}
\end{aligned}
$$

$(3.4)+\left\|\partial_{t} R_{1}(W, S)\right\|_{2}$,

$$
\begin{aligned}
& \left|\partial_{t}\left\|\omega^{m} \nabla \sigma^{\prime}\right\|_{2}\right| \leq C\left\{\|\nabla s\|_{\infty}\left\|\omega^{m} \nabla \sigma^{\prime}\right\|_{2}+\left\|\omega^{m} \nabla s\right\|_{2}\left\|\nabla \sigma^{\prime}\right\|_{\infty}\right. \\
& +\left\|\omega^{m} \nabla \sigma\right\|_{2}\|\nabla S\|_{\infty}+\|\sigma\|_{\infty}\left\|\omega^{m} \nabla^{2} S\right\|_{2} \\
& \left.+t^{-1-\beta(m+1)} a I_{0}\left(\|q\|_{2}\right)\right\}+\left\|\omega^{m} \nabla R_{2}(W, S)\right\|_{2}
\end{aligned}
$$

for $0 \leq m \leq \ell$

$\left|\partial_{t}\left\|\nabla \sigma^{\prime}\right\|_{2}\right| \leq C\left\{\|\nabla s\|_{\infty}\left\|\nabla \sigma^{\prime}\right\|_{2}+\|\nabla \sigma\|_{2}\left(\|\nabla S\|_{\infty}+\left\|\omega^{3 / 2} \nabla S\right\|_{2}\right)\right.$

$$
\left.+t^{-1-\beta} a I_{0}\left(\|q\|_{2}\right)\right\}+\left\|\nabla R_{2}(W, S)\right\|_{2}
$$

$$
\begin{aligned}
& \left\|\partial_{t} \omega^{m} \nabla \sigma^{\prime}\right\|_{2} \leq C\left\{\|s\|_{\infty}\left\|\omega^{m} \nabla^{2} \sigma^{\prime}\right\|_{2}+\left\|\omega^{m} \nabla s\right\|_{2}\left\|\nabla \sigma^{\prime}\right\|_{\infty}\right. \\
& +\left\|\omega^{m} \nabla \sigma\right\|_{2}\|\nabla S\|_{\infty}+\|\sigma\|_{\infty}\left\|\omega^{m} \nabla^{2} S\right\|_{2} \\
& \left.+t^{-1-\beta(m+1)} a I_{0}\left(\|q\|_{2}\right)\right\}+\left\|\omega^{m} \nabla R_{2}(W, S)\right\|_{2}
\end{aligned}
$$

for $0 \leq m \leq \ell-1$,

$$
\begin{gathered}
\left\|\partial_{t} \nabla \sigma^{\prime}\right\|_{2} \leq C\left\{\|\nabla s\|_{\infty}\left\|\nabla \sigma^{\prime}\right\|_{2}+\|s\|_{\infty}\left\|\nabla^{2} \sigma^{\prime}\right\|_{2}+\|\nabla \sigma\|_{2}\right. \\
\left.\times\left(\|\nabla S\|_{\infty}+\left\|\omega^{3 / 2} \nabla S\right\|_{2}\right)+t^{-1-\beta} a I_{0}\left(\|q\|_{2}\right)\right\}+\left\|\nabla R_{2}(W, S)\right\|_{2} \cdot(3.6)_{0}
\end{gathered}
$$

Proof. The estimate (3.2) is essentially (I.6.2) modified by the change $t \rightarrow 1 / t$ and simplified by the fact that $q \in L^{\infty}\left(I, L^{\infty}\right)$ and is proved in the same way. We next prove (3.3). From (1.26) and (2.17) we obtain

$$
\begin{gathered}
\left\|\Delta q^{\prime}\right\|_{2} \leq 2\left\{\left\|\partial_{t} q^{\prime}\right\|_{2}+\left\|Q\left(s, q^{\prime}\right)\right\|_{2}+\|Q(\sigma, w)\|_{2}+C t^{-1} b_{0}\left\|q^{\prime}\right\|_{2}\right. \\
\left.(3.7)+t^{-1}\left\|B_{S}(w, w)\right\|_{\infty}\left\|q^{\prime}\right\|_{2}+t^{-1}\left\|B_{S}(2 W+q, q)\right\|_{2}\|W\|_{\infty}+\left\|R_{1}\right\|_{2}\right\}
\end{gathered}
$$


where $w=W+q$. The terms not containing $q^{\prime}$ have already been estimated in the proof of (3.2). We next estimate

$$
\begin{aligned}
&\left\|Q\left(s, q^{\prime}\right)\right\|_{2} \leq C\left(\|s\|_{\infty}+\|\nabla \cdot s\|_{3}\right)\left\|\nabla q^{\prime}\right\|_{2} \\
& \leq C\left(\|s\|_{\infty}+\|\nabla \cdot s\|_{3}\right)\left\|q^{\prime}\right\|_{2}^{1 / 2}\left\|\Delta q^{\prime}\right\|_{2}^{1 / 2} \\
&\left\|B_{S}(w, w)\right\|_{\infty} \leq C\left\|\nabla B_{S}\right\|_{2}^{1 / 2}\left\|\nabla^{2} B_{S}\right\|_{2}^{1 / 2} \leq C t^{\beta}\left\|\omega^{5 / 2} B_{1}(w, w)\right\|_{2} \\
& \leq C t^{\beta} I_{3 / 2}\left(\left\|\omega^{3 / 2} w\right\|_{2}\|w\|_{\infty}\right) \leq a^{2} t^{\beta}
\end{aligned}
$$

by (2.5) (2.8) and Lemma 2.1. Now (3.3) follows from (3.2) and (3.7)-(3.9).

We next prove (3.4). Taking the time derivative of the equation for $q^{\prime}$ in (1.26), performing a standard $L^{2}$ norm estimate and using the fact that the terms in the RHS containing $\partial_{t} q^{\prime}$ do not contribute to that estimate, we obtain

$$
\begin{aligned}
& \left|\partial_{t}\left\|\partial_{t} q^{\prime}\right\|_{2}\right| \leq\left\|Q\left(\partial_{t} s, q^{\prime}\right)\right\|_{2}+\left\|Q\left(\partial_{t} \sigma, W\right)\right\|_{2}+\left\|Q\left(\sigma, \partial_{t} w\right)\right\|_{2} \\
& +t^{-2}\left\{\left(C b_{0}+\left\|B_{S}(w, w)\right\|_{\infty}\right)\left\|q^{\prime}\right\|_{2}+\left\|B_{S}(2 W+q, q)\right\|_{2}\|W\|_{\infty}\right\} \\
& +t^{-1}\left\{\left\|B_{S}(2 W+q, q)\right\|_{6}\left\|\partial_{t} W\right\|_{3}+\left\|\partial_{t} B_{S}(2 W+q, q)\right\|_{2}\|W\|_{\infty}\right. \\
& \left.+\left\|\partial_{t} B_{S}(w, w)\right\|_{6}\left\|q^{\prime}\right\|_{3}\right\}+\left\|\partial_{t} R_{1}\right\|_{2}
\end{aligned}
$$

We next estimate by Hölder and Sobolev inequalities

$$
\begin{aligned}
\left\|Q\left(\partial_{t} s, q^{\prime}\right)\right\|_{2} & \leq C\left(\left\|\partial_{t} s\right\|_{\infty}+\left\|\partial_{t} \nabla \cdot s\right\|_{3}\right)\left\|\nabla q^{\prime}\right\|_{2} \\
\left\|Q\left(\partial_{t} \sigma, W\right)\right\|_{2} & \leq\left\|\partial_{t} \sigma\right\|_{6}\|\nabla W\|_{3}+\left\|\partial_{t} \nabla \cdot \sigma\right\|_{2}\|W\|_{\infty} \\
& \leq C a\left\|\partial_{t} \nabla \sigma\right\|_{2} \\
\left\|Q\left(\sigma, \partial_{t} W\right)\right\|_{2} & \leq\|\sigma\|_{\infty}\left\|\nabla \partial_{t} W\right\|_{2}+\|\nabla \cdot \sigma\|_{3}\left\|\partial_{t} W\right\|_{6} \\
& \leq C\left(\|\sigma\|_{\infty}+\|\nabla \cdot \sigma\|_{3}\right)\left\|\nabla \partial_{t} W\right\|_{2} .
\end{aligned}
$$

Using in addition (2.5) (2.8), we estimate.

$$
\begin{gathered}
\left\|B_{S}(2 W+q, q)\right\|_{2} \leq C t^{\beta}\left\|\nabla B_{1}(2 W+q, q)\right\|_{2} \leq C a t^{\beta} I_{0}\left(\|q\|_{2}\right) \\
\left\|B_{S}(2 W+q, q)\right\|_{6} \leq C\left\|\nabla B_{1}(2 W+q, q)\right\|_{2} \leq C a I_{0}\left(\|q\|_{2}\right) .
\end{gathered}
$$

We next estimate the terms containing $\partial_{t} B_{S}$. When acting on the cut-off factor $\chi$, the time derivative produces a term

$$
\left(\partial_{t} \chi\left(\xi t^{\beta}\right)\right) B_{1}=\beta t^{-1}(\xi \cdot \nabla \chi)\left(\xi t^{\beta}\right) B_{1}
$$

thereby generating an extra factor $t^{-1}$ and a new cut off field with $\chi$ replaced by $\xi \cdot \nabla \chi$. The corresponding term is easily estimated in the same way as $B_{S}$ and generates terms in the estimates of the same type as those generated by $\partial_{t}$ acting on $t^{-1}$. Omitting those terms, we estimate the remaining contribution of $\partial_{t} B_{S}$ as follows (see (2.6)-(2.8))

$$
\begin{aligned}
& \left\|\partial_{t} B_{S}(2 W+q, q)\right\|_{2} \leq C\left\{t^{\beta} I_{1}\left(\|W+q\|_{\infty}\left\|\partial_{t} q\right\|_{2}\right)\right. \\
& \left.+I_{0}\left(\left\|\partial_{t} W\right\|_{3}\|q\|_{2}\right)\right\} \\
& \leq C\left\{a t^{\beta} I_{1}\left(\left\|\partial_{t} q\right\|_{2}\right)+I_{0}\left(\left\|\partial_{t} W\right\|_{3}\|q\|_{2}\right)\right\},
\end{aligned}
$$




$$
\begin{aligned}
& \left\|\partial_{t} B_{S}(w, w)\right\|_{6} \leq C\left\|\nabla \partial_{t} B_{S}(w, w)\right\|_{2} \\
& \leq C I_{1}\left(\|w\|_{\infty}\left\|\partial_{t} w\right\|_{2}\right) \leq C a I_{1}\left(\left\|\partial_{t} W\right\|_{2}+\left\|\partial_{t} q\right\|_{2}\right) .
\end{aligned}
$$

Substituting (3.9) and (3.11)-(3.17) into (3.10) yields (3.4).

The estimates (3.5) and (3.5) 0 are essentially the estimates (I.6.4) and (I.6.4) $)_{0}$, modified by the change $t \rightarrow 1 / t$ and simplified by the fact that $q \in L^{\infty}\left(I, L^{\infty}\right)$, and are proved in the same way. The estimates (3.6) and (3.6) $)_{0}$ are very similar to (3.5) and $(3.5)_{0}$ and differ therefrom by the fact that the terms $s \cdot \omega^{m} \nabla^{2} \sigma^{\prime}$ and $s \cdot \nabla^{2} \sigma^{\prime}$ cannot be integrated by parts when estimating the $L^{2}$ norm of the relevant time derivative, whereas they can when estimating the derivative of the $L^{2}$ norm.

We also need to estimate the difference of two solutions of the linearized auxiliary system (1.26). Those estimates are given by Lemma I.6.2 in the special case $k=2$, where they become significantly simpler. We restate them in the following lemma.

Lemma 3.2. Let $\beta>0$ and $\ell>3 / 2$. Let $\tau \leq 1$ and $I=(0, \tau]$. Let $B_{0}, W$, $S$ satisfy the assumptions of Lemma 3.1. Let $\left(q_{i}, \sigma_{i}\right)$ and $\left(q_{i}^{\prime}, \sigma_{i}^{\prime}\right), i=1,2$ satisfy the assumptions made on $(q, \sigma)$ and $\left(q^{\prime}, \sigma^{\prime}\right)$ in Lemma 3.1, and in particular let $\left(q_{i}^{\prime}, \sigma_{i}^{\prime}\right)$ be solutions of the system (1.26) corresponding to $\left(q_{i}, \sigma_{i}\right), i=1,2$. Define $\left(q_{ \pm}, \sigma_{ \pm}\right)=(1 / 2)\left(q_{1} \pm q_{2}, \sigma_{1} \pm \sigma_{2}\right)$ and $\left(q_{ \pm}^{\prime}, \sigma_{ \pm}^{\prime}\right)=(1 / 2)\left(q_{1}^{\prime} \pm q_{2}^{\prime}, \sigma_{1}^{\prime} \pm \sigma_{2}^{\prime}\right)$. Then the following estimates hold for all $t \in I$

$$
\left|\partial_{t}\left\|q_{-}^{\prime}\right\|_{2}\right| \leq C\left\{a\left\|\nabla \sigma_{-}\right\|_{2}+a^{2} t^{-1+\beta} I_{0}\left(\left\|q_{-}\right\|_{2}\right)\right\},
$$

$\left|\partial_{t}\left\|\nabla \sigma_{-}^{\prime}\right\|_{2}\right| \leq C\left\{\left\|\nabla s_{+}\right\|_{\infty}\left\|\nabla \sigma_{-}^{\prime}\right\|_{2}+\left(\left\|\nabla s_{+}^{\prime}\right\|_{\infty}+\left\|\nabla^{2} s_{+}^{\prime}\right\|_{3}\right)\left\|\nabla \sigma_{-}\right\|_{2}\right.$

$$
\left.+a t^{-1-\beta} I_{0}\left(\left\|q_{-}\right\|_{2}\right)\right\},
$$

where $s_{+}=S+\sigma_{+}, s_{+}^{\prime}=S+\sigma_{+}^{\prime}$.

We can now solve the Cauchy problem at zero for the auxiliary system (1.23) under suitable boundedness properties of $\left(B_{0}, W, S\right)$ and suitable vanishing properties of the remainders at zero. This is the first main result of this section. It corresponds to Proposition I.6.3, part (2) and to Proposition II.3.1.

Proposition 3.1 Let $0<\beta<1, \ell>3 / 2$ and $\lambda_{0}>1 \vee \beta(\ell+1)$. Let $B_{0} \in$ $\mathcal{C}^{1}\left((0,1], L^{\infty}\right)$ satisfy the estimate (2.17) for $r=\infty$ and $j=0,1$. Let $(W, \nabla S) \in$ $\mathcal{C}\left((0,1], H^{2} \oplus H^{\ell+1}\right) \cap \mathcal{C}^{1}\left((0,1], H^{1} \oplus H^{\ell-1}\right)$, let $R_{1} \in \mathcal{C}^{1}\left((0,1], L^{2}\right)$ and $\nabla R_{2} \in$ $\mathcal{C}\left((0,1], H^{\ell}\right)$. Let $W, S, R_{1}, R_{2}$ satisfy the following estimates for all $t \in(0,1]$ :

$$
\begin{gathered}
\|W\|_{\infty} \vee|W|_{3 / 2} \leq a \\
\left|\partial_{t} W\right|_{1} \leq a_{1} t^{-1 / 2} \\
\left\|\omega^{m} \nabla S\right\|_{2} \leq b\left(|\ell n t|+t^{-\beta(m-2)}\right) \quad \text { for } 0 \leq m \leq \ell+1, \\
\left\|\partial_{t} S\right\|_{\infty} \vee\left\|\partial_{t} \nabla S\right\|_{3} \leq b_{1} t^{-1} \\
\left\|\partial_{t}^{j} R_{1}\right\|_{2} \leq r_{1} t^{\lambda_{0}-1-j} \quad \text { for } j=0,1,
\end{gathered}
$$




$$
\left\|\omega^{m} \nabla R_{2}\right\|_{2} \leq r_{2} t^{\lambda_{0}-1-\beta(m+1)} \quad \text { for } 0 \leq m \leq \ell .
$$

Then there exists $\tau, 0<\tau \leq 1$ and positive constants $Y_{0}, Y_{1}, Y, Z$, depending on $\beta, \ell, \lambda_{0}, b_{0}, a, a_{1}, b, b_{1}, r_{1}, r_{2}$ such that the auxiliary system (1.20) has a unique solution $(w, s)$ such that $(w, \nabla s) \in \mathcal{C}\left(I, H^{2} \oplus H^{\ell}\right) \cap \mathcal{C}^{1}\left(I, L^{2} \oplus H^{\ell-1}\right)$, where $I=(0, \tau]$, and such that $(q, \sigma) \equiv(w-W, s-S)$ satisfies the estimates

$$
\begin{gathered}
\|q\|_{2} \leq Y_{0} t^{\lambda_{0}} \\
\left\|\partial_{t} q\right\|_{2} \leq Y_{1} t^{\lambda_{0}-1} \\
\|\Delta q\|_{2} \leq Y t^{\lambda_{0}-1} \\
\left\|\omega^{m} \nabla \sigma\right\|_{2} \leq Z t^{\lambda_{0}-\beta(m+1)} \quad \text { for } 0 \leq m \leq \ell, \\
\left\|\omega^{m} \nabla \partial_{t} \sigma\right\|_{2} \leq Z t^{\lambda_{0}-1-\beta(m+1)} \quad \text { for } 0 \leq m \leq \ell-1
\end{gathered}
$$

for all $t \in I$.

Sketch of proof. The proof follows closely that of Proposition I.6.3, part (2). We first take $\tau, 0<\tau \leq 1$, and $(q, \sigma)$ satisfying the conditions of the proposition and in particular the estimates (3.26)-(3.30) for all $t \in I=(0, \tau]$. We then take $t_{0}$, $0<t_{0}<\tau$, and solve the Cauchy problem for the linearized system (1.26) with initial condition $\left(q^{\prime}, \sigma^{\prime}\right)\left(t_{0}\right)=0$, by the use of Proposition I.6.1 with $k=2$ and of Proposition 3.2 in [4]. Let $\left(q_{t_{0}}^{\prime}, \sigma_{t_{0}}^{\prime}\right)$ be the solution thereby obtained. Using Lemma 3.1, we then show that $\left(q_{t_{0}}^{\prime}, \sigma_{t_{0}}^{\prime}\right)$ satisfies estimates similar to (3.26)-(3.30) with constants $Y_{0}^{\prime}, Y_{1}^{\prime}, Y^{\prime}, Z^{\prime}$, uniformly in $t_{0}$ for $t \in\left[t_{0}, \tau\right]$. Using Lemma 3.2, we take the limit $t_{0} \rightarrow 0$ of $\left(q_{t_{0}}^{\prime}, \sigma_{t_{0}}^{\prime}\right)$, thereby obtaining a solution $\left(q^{\prime}, \sigma^{\prime}\right)$ of the system $(1.26)$ in $(0, \tau]$ satisfying the same estimates in that interval. We finally prove that for sufficiently small $\tau$, the map $(q, \sigma) \rightarrow\left(q^{\prime}, \sigma^{\prime}\right)$ thereby defined is a contraction in the norms of Lemma 3.2 on a suitable bounded set defined by the conditions (3.26)-(3.30) for suitably chosen $Y_{0}, Y_{1}, Y, Z$. The abstract arguments are the same as in Proposition I.6.2 and I.6.3, part (2), and the only difference lies in the estimates of $\left(q^{\prime}, \sigma^{\prime}\right)$ for given $(q, \sigma)$, which now involve a time derivative instead of space derivatives only. In the remaining parts of this sketch, we concentrate on the derivation of those estimates. We first estimate $\left(q_{t_{0}}^{\prime}, \sigma_{t_{0}}^{\prime}\right)$ defined above, assuming that $(q, \sigma)$ satisfies (3.26)-(3.30). Omitting the subscript $t_{0}$ for brevity, we define

$$
\begin{gathered}
\left\{\begin{array}{l}
y_{0}^{\prime}=\left\|q^{\prime}\right\|_{2}, \quad y_{1}^{\prime}=\left\|\partial_{t} q^{\prime}\right\|_{2}, \quad y^{\prime}=\left\|\Delta q^{\prime}\right\|_{2} \\
z_{m}^{\prime}=\left\|\omega^{m} \nabla \sigma^{\prime}\right\|_{2} \quad \text { for } 0 \leq m \leq \ell,
\end{array}\right. \\
\left\{\begin{array}{l}
Y_{0}^{\prime}=\operatorname{Sup}_{t \in I_{0}} t^{-\lambda_{0}} y_{0}^{\prime}, \quad Y_{1}^{\prime}=\operatorname{Sup}_{t \in I_{0}} t^{1-\lambda_{0}} y_{1}^{\prime}, \quad Y^{\prime}=\operatorname{Sup}_{t \in I_{0}} t^{1-\lambda_{0}} y^{\prime} \\
Z^{\prime}=\operatorname{Sup}_{0 \leq m \leq \ell} Z_{m}^{\prime}, \quad Z_{m}^{\prime}=\operatorname{Sup}_{t \in I_{0}} t^{\beta(m+1)-\lambda_{0}} z_{m}^{\prime},
\end{array}\right.
\end{gathered}
$$

where $I_{0}=\left[t_{0}, \tau\right]$. We first estimate $Y_{0}^{\prime}$. From (3.2) (3.24) (3.26) (3.29) we obtain

$$
\left|\partial_{t} y_{0}^{\prime}\right| \leq C\left(a Z t^{\lambda_{0}-\beta}+a^{2} Y_{0} t^{\lambda_{0}-1+\beta}\right)+r_{1} t^{\lambda_{0}-1}=N(t) t^{\lambda_{0}-1}
$$

where

$$
N(t)=C\left(a Z t^{1-\beta}+a^{2} Y_{0} t^{\beta}\right)+r_{1}
$$


and therefore by integration

$$
y_{0}^{\prime} \leq \int_{t_{0}}^{t} d t^{\prime} t^{\prime \lambda_{0}-1} N\left(t^{\prime}\right) \leq N(t) \lambda_{0}^{-1} t^{\lambda_{0}}<N(t) t^{\lambda_{0}}
$$

so that

$$
Y_{0}^{\prime} \leq N(\tau) .
$$

We next estimate $Y^{\prime}$. From (3.2) (3.3) (3.22) (3.29) (3.33) (3.35) we obtain

$$
y^{\prime} \leq C\left\{y_{1}^{\prime}+N(t)\left(1+b_{0}+a^{2} t^{\beta}+t\left(b^{2}|\ln t|^{2}+Z^{2} t^{2 \lambda_{0}-3 \beta}\right)\right) t^{\lambda_{0}-1}\right\}
$$

and therefore

$$
Y^{\prime} \leq C\left\{Y_{1}^{\prime}+N(\tau)\left(1+b_{0}+a^{2} \tau^{\beta}+b^{2} \tau|\ell n \tau|^{2}+Z^{2} \tau^{2 \lambda_{0}+1-3 \beta}\right)\right\} .
$$

We next estimate $Y_{1}^{\prime}$. From (3.4) (3.21) (3.23) (3.24) (3.26) (3.27) (3.30) and the definitions, we obtain

$$
\begin{gathered}
\left|\partial_{t} y_{1}^{\prime}\right| \leq C\left\{\left(b_{1} t^{\lambda_{0}-3 / 2}+Z t^{2 \lambda_{0}-3(1+\beta) / 2}\right)\left(Y_{0}^{\prime} Y^{\prime}\right)^{1 / 2}\right. \\
+Z\left(a t^{\lambda_{0}-1-\beta}+a_{1} t^{\lambda_{0}-1 / 2-3 \beta / 2}\right)+b_{0} Y_{0}^{\prime} t^{\lambda_{0}-2} \\
+a^{2}\left(Y_{0}^{\prime}+Y_{0}+Y_{1}\right) t^{\lambda_{0}-2+\beta}+a a_{1} Y_{0} t^{\lambda_{0}-3 / 2} \\
\left.+a\left(a_{1} t^{\lambda_{0}-7 / 4}+Y_{1} t^{2 \lambda_{0}-9 / 4}\right) Y_{0}^{\prime 3 / 4} Y^{\prime / 4}\right\}+r_{1} t^{\lambda_{0}-2} .
\end{gathered}
$$

The initial condition for $y_{1}^{\prime}$ at $t=t_{0}$ is estimated by

$$
y_{1}^{\prime}\left(t_{0}\right) \leq N\left(t_{0}\right) t_{0}^{\lambda_{0}-1} \leq N\left(t_{0}\right) t^{\lambda_{0}-1}
$$

for all $t \in I_{0}$. Integrating (3.37) with initial condition (3.38) and using the definitions yields

$$
\begin{aligned}
& Y_{1}^{\prime} \leq C\left\{\left(b_{1} \tau^{1 / 2}+Z \tau^{\lambda_{0}+(1-3 \beta) / 2}\right)\left(Y_{0}^{\prime} Y^{\prime}\right)^{1 / 2}+a Z \tau^{1-\beta}\right. \\
&+a_{1} Z \tau^{3(1-\beta) / 2}+b_{0} Y_{0}^{\prime}+a^{2}\left(Y_{0}^{\prime}+Y_{0}+Y_{1}\right) \tau^{\beta}+a a_{1} Y_{0} \tau^{1 / 2} \\
&\left.+a\left(a_{1} \tau^{1 / 4}+Y_{1} \tau^{\lambda_{0}-1 / 4}\right) Y_{0}^{\prime 3 / 4} Y^{\prime / 4}+r_{1}\right\} .
\end{aligned}
$$

We next estimate $Z^{\prime}$. It is sufficient to estimate $Z_{0}^{\prime}$ and $Z_{\ell}^{\prime}$. The general case follows by interpolation. The estimates proceed exactly as in I, with minor differences due to the slightly different assumption made on $S$ and to the simplification produced by the fact that now $q \in L^{\infty}\left(I, L^{\infty}\right)$. We obtain (see (I.6.68) and (I.6.71))

$$
\begin{gathered}
Z_{0}^{\prime} \leq C \exp (C(b \tau(1-\ell n \tau)+Z \tau))\left\{b Z \tau(1-\ell n \tau)+a Y_{0}+r_{2}\right\} \\
Z_{\ell}^{\prime} \leq C \exp (C(b \tau(1-\ell n \tau)+Z \tau))\left\{b \tau(1-\ell n \tau)\left(Z+Z_{0}^{\prime}\right)+\tau Z Z_{0}^{\prime}+a Y_{0}+r_{2}\right\} .
\end{gathered}
$$

From (3.35) (3.36) (3.39) (3.40) (3.41) and from similar easy estimates of $\left\|\partial_{t} \omega^{m} \nabla \sigma^{\prime}\right\|_{2}$ that follow from (3.6) $(3.6)_{0}$ and from the previous estimates of $\left\|\omega^{m} \nabla \sigma^{\prime}\right\|_{2}$, it follows that $\left(q_{t_{0}}^{\prime}, \sigma_{t_{0}}^{\prime}\right)$ satisfies estimates similar to (3.26) -(3.30) with constants $Y_{0}^{\prime}$, $Y_{1}^{\prime}, Y^{\prime}$ and $Z^{\prime}$ satisfying the estimates just derived, for $t \in\left[t_{0}, \tau\right]$, uniformly in $t_{0}$. From there on, the proof proceeds by straightforward modifications of those of Propositions I.6.2 and I.6.3, part (2). Using Lemma 3.2, one takes the limit $t_{0} \rightarrow 0$ of $\left(q_{t_{0}}^{\prime}, \sigma_{t_{0}}^{\prime}\right)$, thereby obtaining a solution $\left(q^{\prime}, \sigma^{\prime}\right)$ of the linearized system (1.26) in $I=(0, \tau]$ with the same regularity and satisfying the same estimates for all $t \in I$. This defines a map $\phi:(q, \sigma) \rightarrow\left(q^{\prime}, \sigma^{\prime}\right)$ and the previous estimates prove that this map is bounded in the norm corresponding to (3.26)-(3.30). One finally shows that 
$\phi$ is a contraction on the set $\mathcal{R}$ defined by (3.26)-(3.30) for the norm corresponding to (3.26) and (3.29) with $m=0$ for suitably chosen $Y_{0}, Y_{1}, Y, Z$ and sufficiently small $\tau$. The proof is a minor variant of that of Proposition I.6.3, part (2) and will be omitted.

Remark 3.1. Stronger uniqueness results for the system (1.23) than stated in Proposition 3.1 and stronger uniqueness results for the system (1.20) regardless on whether $(w, s)$ is an approximation of some given $(W, S)$ can be obtained by a minor variant of Proposition I.4.2. For instance if $\left(w_{1}, s_{1}\right)$ and $\left(w_{2}, s_{2}\right)$ are two solutions of the system $(1.20)$ in $I=(0, \tau]$ satisfying the regularity properties stated for $(w, s)$ in Proposition 3.1 and the estimates

$$
\begin{gathered}
\left\|w_{i}\right\|_{\infty} \vee\left|w_{i}\right|_{3 / 2} \leq a \\
\left\|\omega^{m} \nabla s_{i}\right\|_{2} \leq b|\ell n t| \quad \text { for } 0 \leq m \leq \ell
\end{gathered}
$$

for all $t \in I$ or equivalently in a neighborhood of zero and if $t^{-\beta}\left\|w_{1}-w_{2}\right\|_{2}$ and $\left\|\nabla\left(s_{1}-s_{2}\right)\right\|_{2}$ tend to zero when $t \rightarrow 0$, then $\left(w_{1}, s_{1}\right)=\left(w_{2}, s_{2}\right)$. This can be proved as in Proposition I.4.2, part (3), starting from a minor variant of Lemma 3.2.

We now turn to the construction of approximate solutions $(W, S)$ of the system (1.20) satisfying the assumptions of Proposition 3.1 and in particular the estimates (3.20)-(3.25) of $W, S$ and of the remainders. As in II, we take for $(W, S)$ the second order approximate solution of the system (1.20) in an iterative scheme not taking $B_{0}$ into account (previously used in I), supplemented by an additional term in $W$ in order to partly cancel $B_{0 S} W$ in $R_{1}(W, S)$. Thus we define

$$
W=w_{0}+w_{1}+w_{2} \equiv W_{1}+w_{2}, \quad S=s_{0}+s_{1}
$$

where (up to the change of $t$ into $1 / t) w_{0}, w_{1}, s_{0}, s_{1}$ are the same as in I except for a simplification of $w_{1}$ namely

$$
\begin{gathered}
w_{0}=U(t) w_{+} \\
s_{0}(t)=\int_{t}^{1} d t^{\prime} t^{\prime-1} \nabla B_{L}\left(w_{0}\left(t^{\prime}\right), w_{0}\left(t^{\prime}\right)\right) \\
w_{1}(t)=\int_{0}^{t} d t^{\prime} Q\left(s_{0}\left(t^{\prime}\right), w_{0}\left(t^{\prime}\right)\right) \\
s_{1}(t)=\int_{0}^{t} d t^{\prime}\left(s_{0}\left(t^{\prime}\right) \cdot \nabla s_{0}\left(t^{\prime}\right)-2 t^{\prime-1} \nabla B_{L}\left(w_{0}\left(t^{\prime}\right), w_{1}\left(t^{\prime}\right)\right)\right)
\end{gathered}
$$

while $w_{2}$ is the same as in II, namely

$$
w_{2}=h w_{0} \quad, \quad h=-2 t^{-1} \Delta^{-1} B_{0 S} .
$$

With that choice, the remainders become

$$
\begin{gathered}
R_{1}(W, S)=(1 / 2) \Delta w_{1}+i\left(\partial_{t} h\right) w_{0}+(\nabla h) \cdot \nabla w_{0}-i Q\left(s_{0}, w_{1}+w_{2}\right) \\
-i Q\left(s_{1}, W\right)+t^{-1} B_{0 S}\left(w_{1}+w_{2}\right)+t^{-1} B_{S}(W, W) W \\
R_{2}(W, S)=R_{20}(W, S)+R_{2 \nu}(W, S)
\end{gathered}
$$

where

$$
\begin{gathered}
R_{20}(W, S)=-\left(s_{0} \cdot \nabla s_{1}+s_{1} \cdot \nabla s_{0}+s_{1} \cdot \nabla s_{1}\right)+t^{-1} \nabla B_{L}\left(w_{1}, w_{1}\right) \\
R_{2 \nu}(W, S)=t^{-1} \nabla B_{0 L}+t^{-1} \nabla B_{L}\left(W+W_{1}, w_{2}\right) .
\end{gathered}
$$


The terms not involving $h$ or $w_{2}$ have already been estimated in I. Up to appropriate minor changes and additions, the following lemma is basically Lemma I.7.1 or Lemma II.3.2.

Lemma 3.3. Let $0<\beta<1, k_{+} \geq 3, w_{+} \in H^{k_{+}}$and $a_{+}=\left|w_{+}\right| k_{+}$. Then the following estimates hold for all $t, 0<t \leq 1$ :

$$
\begin{aligned}
& \left|\partial_{t} w_{0}\right|_{k_{+}-2} \leq\left|w_{0}\right|_{k_{+}}=a_{+} \\
& \left\|\omega^{m} s_{0}\right\|_{2} \leq C a_{+}^{2}\left(|\ln t|+t^{-\beta\left(m-k_{+}\right)}\right) \quad \text { for } m \geq 0 \text {, } \\
& \left\|\omega^{m} \partial_{t} s_{0}\right\|_{2} \leq C a_{+}^{2} t^{-1-\beta\left(m-k_{+}\right)_{+}} \quad \text { for } m \geq 0, \\
& \left|w_{1}\right|_{k_{+}-1} \leq C a_{+}^{3} t(1-\ln t) \\
& \left|\partial_{t} w_{1}\right|_{k_{+}-1} \leq C a_{+}^{3}|\ln t| \\
& \left\|\omega^{m} s_{1}\right\|_{2} \leq C a_{+}^{4} t\left\{(1-\ell n t)^{2}+(1-\ell n t) t^{-\beta\left(m+1-k_{+}\right)}\right\} \\
& \text {for } m \geq 0, \beta\left(m+1-k_{+}\right)<1 \text {, } \\
& \left\|\omega^{m} \partial_{t} s_{1}\right\|_{2} \leq C a_{+}^{4}\left\{(1-\ell n t)^{2}+(1-\ell n t) t^{-\beta\left(m+1-k_{+}\right)}\right\} \\
& \text {for } m \geq 0, \beta\left(m+1-k_{+}\right)<1, \\
& \left\|\omega^{m} R_{20}(W, S)\right\|_{2} \leq C\left(a_{+}\right) t\left\{(1-\ln t)^{3}+(1-\ln t)^{2} t^{-\beta\left(m+2-k_{+}\right)}\right\} \\
& \text {for } m \geq 0, \beta\left(m+2-k_{+}\right)<1 \text {. }
\end{aligned}
$$

In order to complete the estimates of $W$ and of the remainders, we need some estimates of $B_{0 L}$ and of $h$. Those estimates are the same as in II, supplemented by an estimate of $\partial_{t}^{2} h$. They require some restrictions on the behaviour of $\left(F A_{+}, F \dot{A}_{+}\right)$at $\xi=0$, which we impose as in II in a dilation homogeneous way in terms of quantities which have the same scaling properties as $\left\|A_{+} ; \dot{H}^{-3 / 2-\mu}\right\|$ and $\left\|\dot{A}_{+} ; \dot{H}^{-5 / 2-\mu}\right\|$ for some $\mu \in(-1,1)$. The following lemma is a minor extension of Lemma II.3.4 and its proof will be omitted.

Lemma 3.4. Let $0<\beta<1$ and $-1<\mu<1$. Let $\left(A_{+}, \dot{A}_{+}\right)$satisfy the conditions

$$
A_{+}, x A_{+}, x^{2} A_{+} \in H^{1} \quad, \quad \dot{A}_{+}, x \dot{A}_{+}, x^{2} \dot{A}_{+} \in L^{2}
$$

$x^{2} A_{+} \in \dot{H}^{1 / 2-\mu}, x A_{+}, x^{2} \dot{A}_{+} \in \dot{H}^{-1 / 2-\mu}, A_{+}, x \dot{A}_{+} \in \dot{H}^{-3 / 2-\mu}, \dot{A}_{+} \in \dot{H}^{-5 / 2-\mu}$.

Let $B_{0 L}$ and $h$ be defined by (1.17) and (3.47). Then the following estimates hold:

$$
\left\|\omega^{m} B_{0 L}\right\|_{2} \leq C t^{2+\mu-\beta(m+3 / 2+\mu)}\left(\left\|A_{+} ; \dot{H}^{-3 / 2-\mu}\right\|+\left\|\dot{A}_{+} ; \dot{H}^{-5 / 2-\mu}\right\|\right)
$$

for all $m \geq 0$,

$\left\|\omega^{m} \partial_{t}^{j} h\right\|_{2} \leq C t^{3 / 2-m-j} 1_{m} \sum_{0 \leq j^{\prime} \leq j}\left(\left\||x|^{j^{\prime}} A_{+} ; \dot{H}^{\rho+j^{\prime}}\right\|+\left\||x|^{j^{\prime}} \dot{A}_{+} ; \dot{H}^{\rho+j^{\prime}-1}\right\|\right)$

for $j=0,1,2$ and $m \leq 3-j$, where

$$
1_{m}=1 \vee t^{(1-\beta)(m-1 / 2+\mu)}
$$


and

$$
\begin{gathered}
\rho=(m-2) \vee(-3 / 2-\mu), \\
\|h\|_{\infty} \leq C\left(A_{+}, \dot{A}_{+}\right)
\end{gathered}
$$

where the constant depends on $\left(A_{+}, \dot{A}_{+}\right)$through the norms in (3.60) (3.61) $)_{\mu}$ not involving $x$.

We can now state the final result on the Cauchy problem at zero for the auxiliary system (1.20).

Proposition 3.2. Let $0<\beta<3 / 5, \ell>3 / 2$ and $1 \vee \beta(\ell+1)<\lambda_{0}<3 / 2$. Let $k_{+}$ and $\mu$ satisfy

$$
\begin{gathered}
k_{+} \geq \lambda_{0}+2 \quad, \quad \beta\left(k_{+}+1\right) \geq \lambda_{0}, \\
\mu \geq(1-\beta)^{-1}\left\{\lambda_{0}-1-\beta / 2+(3 \beta-1) \vee 0\right\} \quad(>-1 / 4) .
\end{gathered}
$$

Let $w_{+} \in H^{k_{+}}$, and let $\left(A_{+}, \dot{A}_{+}\right)$satisfy (2.16) (3.60) (3.61) $)_{\mu}$. Let $(W, S)$ be defined by (3.42)-(3.47). Then there exists $\tau, 0<\tau \leq 1$ such that the auxiliary system (1.20) has a unique solution $(w, s)$ such that $(w, \nabla s) \in \mathcal{C}\left(I, H^{2} \oplus H^{\ell}\right) \cap$ $\mathcal{C}^{1}\left(I, L^{2} \oplus H^{\ell-1}\right)$, where $I=(0, \tau]$, and satisfying

$$
\begin{gathered}
\|w(t)-W(t)\|_{2} \leq C\left(a_{+}, A_{+}, \dot{A}_{+}\right) t^{\lambda_{0}} \\
\left\|\partial_{t}(w(t)-W(t))\right\|_{2} \vee\|\Delta(w(t)-W(t))\|_{2} \leq C\left(a_{+}, A_{+}, \dot{A}_{+}\right) t^{\lambda_{0}-1} \\
\left\|\omega^{m}(s(t)-S(t))\right\|_{2} \leq C\left(a_{+}, A_{+}, \dot{A}_{+}\right) t^{\lambda_{0}-\beta m} \text { for } 0 \leq m \leq \ell+1 \\
\left\|\omega^{m} \partial_{t}(s(t)-S(t))\right\|_{2} \leq C\left(a_{+}, A_{+}, \dot{A}_{+}\right) t^{\lambda_{0}-1-\beta m} \text { for } 0 \leq m \leq \ell
\end{gathered}
$$

for all $t \in(0, \tau]$, where $a_{+}=\left|w_{+}\right|_{k_{+}}$, and the constants $C\left(a_{+}, A_{+}, \dot{A}_{+}\right)$depend on $\left(A_{+}, \dot{A}_{+}\right)$through the norms associated with (2.16) (3.60) (3.61) $)_{\mu}$.

Proof. Proposition 3.2 follows from Proposition 3.1 and from the fact that $(W, S)$ defined by (3.42)-(3.47) satisfies the assumptions of the latter under the assumptions made here, and in particular satisfies the estimates (3.20)-(3.25). The regularity properties are easily seen to hold and we concentrate on the estimates. The estimates (3.20) (3.21) as regards $W_{1}=w_{0}+w_{1}$ and the estimates (3.22) (3.23) follow immediately from Lemma 3.3. We estimate $w_{2}$ by Lemma 3.4 and possibly Lemmas 2.1 and 2.2 , namely

$$
\begin{gathered}
\left\|w_{2}\right\|_{2} \leq\|h\|_{2}\left\|w_{0}\right\|_{\infty} \leq C t^{3 / 2} 1_{0} \\
\left\|\omega^{m} w_{2}\right\|_{2} \leq C\left(\left\|\omega^{m} h\right\|_{2}\left\|w_{0}\right\|_{\infty}+\|h\|_{r}\left\|\omega^{m} w_{0}\right\|_{3 / \delta}\right)
\end{gathered}
$$

by Lemma 2.2 with $2<r<\infty$, so that $0<\delta \equiv 3 / 2-3 / r<3 / 2$,

$$
\cdots \leq C\left(\left\|\omega^{m} h\right\|_{2}\left\|w_{0}\right\|_{\infty}+\left\|\omega^{\delta} h\right\|_{2}\left\|\omega^{m+3 / 2-\delta} w_{0}\right\|_{2}\right)
$$

by Lemma 2.1,

$$
\cdots \leq C\left(t^{3 / 2-m} 1_{m}+t^{3 / 2-\delta} 1_{\delta}\right) \leq C t^{3 / 2-m} 1_{m}
$$

for $0<m<3$ with $k_{+} \geq 3$ by Lemma 3.4, provided $m-3 / 2 \leq \delta \leq m$. One can take for instance $\delta=m / 2$. 
We next estimate

$$
\begin{gathered}
\left\|\partial_{t} w_{2}\right\|_{2} \leq\left\|\partial_{t} h\right\|_{2}\left\|w_{0}\right\|_{\infty}+\|h\|_{3}\left\|\Delta w_{0}\right\|_{6} \leq C t^{1 / 2} 1_{0} \\
\left\|\partial_{t} \nabla w_{2}\right\|_{2} \leq\left\|\partial_{t} \nabla h\right\|_{2}\left\|w_{0}\right\|_{\infty}+\left\|\partial_{t} h\right\|_{2}\left\|\nabla w_{0}\right\|_{\infty} \\
+\|\nabla h\|_{3}\left\|\Delta w_{0}\right\|_{6}+\|h\|_{\infty}\left\|\nabla \Delta w_{0}\right\|_{2} \leq C t^{-1 / 2}
\end{gathered}
$$

which completes the proof of (3.20) (3.21). We now turn to the remainders and to the proof of (3.24) (3.25). The $L^{2}$ estimate of $R_{1}$ has already been proved in II but we sketch its proof again because (i) it is simpler in the present case and (ii) it is the starting point to prove the estimate of $\partial_{t} R_{1}$, which is new. We estimate

$$
\begin{gathered}
\left\|R_{1}\right\|_{2} \leq\left\|\Delta w_{1}\right\|_{2}+\left\|\partial_{t} h\right\|_{2}\left\|w_{0}\right\|_{\infty}+\|\nabla h\|_{2}\left\|\nabla w_{0}\right\|_{\infty} \\
+\left(\left\|s_{0}\right\|_{\infty}+C\left\|\nabla \cdot s_{0}\right\|_{3}\right)\left(\left\|\nabla w_{1}\right\|_{2}+\left\|\nabla w_{2}\right\|_{2}\right) \\
+\left(\left\|s_{1}\right\|_{\infty}+C\left\|\nabla \cdot s_{1}\right\|_{3}\right)\|\nabla W\|_{2}+t^{-1}\left\|B_{0}\right\|_{2}\left\|w_{1}\right\|_{\infty} \\
+t^{-1}\left\|B_{0}\right\|_{\infty}\left\|w_{2}\right\|_{2}+t^{-1}\|W\|_{\infty}\left\|B_{S}(W, W)\right\|_{2} .
\end{gathered}
$$

We shall need a slightly more general estimate of $B_{S}(W, W)$ than required for (3.77). We separate

$$
B_{S}(W, W)=B_{S}\left(w_{0}, w_{0}\right)+B_{S}\left(w_{1}, w_{0}+W_{1}\right)+B_{S}\left(w_{2}, W+W_{1}\right)
$$

and we estimate

$$
\begin{gathered}
\left\|\omega^{m} B_{S}\left(w_{0}, w_{0}\right)\right\| \leq C t^{\beta\left(k_{+}+1-m\right)} I_{k_{+}}\left(\left\|\omega^{k_{+}} w_{0}\right\|_{2}\left\|w_{0}\right\|_{\infty}\right) \\
\leq C a_{+}^{2} t^{\beta\left(k_{+}+1-m\right)}
\end{gathered}
$$

for $0 \leq m \leq k_{+}+1$, by (2.5) (2.8) and Lemma 2.2,

$$
\begin{gathered}
\left\|\omega^{m} B_{S}\left(w_{1}, w_{0}+W_{1}\right)\right\|_{2} \leq C t^{\beta\left(k_{+}-m\right)} I_{k_{+}-1}\left(\left\|\omega^{k_{+}-1} w_{1}\right\|_{2}\right. \\
\left.\times\left(\left\|w_{0}\right\|_{\infty}+\left\|w_{1}\right\|_{\infty}\right)+\left\|\omega^{k_{+}} w_{0}\right\|_{2}\left\|w_{1}\right\|_{3}\right) \\
\leq C\left(a_{+}\right) t^{\beta\left(k_{+}-m\right)+1}(1-\ell n t)
\end{gathered}
$$

for $0 \leq m \leq k_{+}$, by (2.5) (2.8), Lemma 2.2 and (3.55),

$$
\begin{aligned}
\left\|\omega^{m} B_{S}\left(w_{2}, W+W_{1}\right)\right\|_{2} \leq & C t^{\beta(1-m)} I_{0}\left(\left\|w_{2}\right\|_{2}\left(\left\|w_{2}\right\|_{\infty}+\left\|W_{1}\right\|_{\infty}\right)\right) \\
& \leq C t^{\beta(1-m)+3 / 2} 1_{0}
\end{aligned}
$$

for $0 \leq m \leq 1$, by (2.5) (2.8) (3.73), so that

$$
\left\|\omega^{m} B_{S}(W, W)\right\|_{2} \leq C\left(t^{\beta\left(k_{+}+1-m\right)}+t^{\beta(1-m)+3 / 2} 1_{0}\right)
$$

for $0 \leq m \leq 1$. From (3.77) (2.17), from Lemmas 3.3 and 3.4 and from (3.82) with $m=0$, it follows that

$$
\left\|R_{1}\right\|_{2} \leq C\left\{t^{1 / 2}\left(|\ln t|+1_{0}\right)+t^{\beta\left(k_{+}+1\right)-1}\right\}
$$

so that $R_{1}$ satisfies (3.24) with $j=0$ provided $\lambda_{0}<3 / 2, \lambda_{0} \leq \beta\left(k_{+}+1\right)$ and

$$
\lambda_{0} \leq 1+\mu-\beta(\mu-1 / 2)
$$

which is part of (3.68). We next estimate $\partial_{t} R_{1}$ in $L^{2}$. The estimate of $\partial_{t} R_{1}$ is obtained from (3.77) by inserting an additional $\partial_{t}$ at all possible places and possibly changing the exponents in the application of the Hölder inequality. If no such change is required, it follows from (2.17) and from Lemmas 3.3 and 3.4 that the estimate gets worse by at most one power of $t$ so that all such terms satisfy the $j=1$ case of (3.24). It is therefore sufficient to consider only the terms where the 
Hölder exponents have to be changed. This comes about mostly as a consequence of the fact that $\partial_{t} w_{0} \sim \Delta w_{0}$ has limited regularity, and in particular is in general not in $L^{\infty}$ under the assumption made on $k_{+}$. Thus we estimate

$$
\begin{gathered}
\left\|\partial_{t} R_{1}\right\|_{2} \leq\left\|\partial_{t} h\right\|_{3}\left\|\partial_{t} w_{0}\right\|_{6}+C\|\nabla h\|_{r}\left|\nabla \partial_{t} w_{0}\right|_{k_{+}-3} \\
+t^{-1}\left\{\left\|\partial_{t} W\right\|_{6}\left\|B_{S}(W, W)\right\|_{3}+2\|W\|_{\infty} \| \widetilde{B}_{S}\left(\partial_{t} w_{0}, W \|_{2}\right\}\right. \\
+ \text { other terms }
\end{gathered}
$$

where $3 / r=k_{+}-3$ for $3<k_{+}<9 / 2$, the only dangerous case, and $r=2$ for $k_{+}>9 / 2$, and where $\widetilde{B}$ is defined by $(2.7)$. We next estimate

$$
\begin{gathered}
\left\|\partial_{t} h\right\|_{3}\left\|\partial_{t} w_{0}\right\|_{6} \leq C\left\|\omega^{1 / 2} \partial_{t} h\right\|_{2}\left\|\nabla \Delta w_{0}\right\|_{2} \\
\leq C 1_{1 / 2} \leq C t^{\lambda_{0}-2}
\end{gathered}
$$

for $k_{+} \geq 3$

$$
\begin{gathered}
\|\nabla h\|_{r}\left|\nabla \partial_{t} w_{0}\right|_{k_{+}-3} \leq\left\|\omega^{11 / 2-k_{+}} h\right\|_{2}\left|w_{0}\right|_{k_{+}} \\
\leq C t^{k_{+}-4} \leq C t^{\lambda_{0}-2}
\end{gathered}
$$

for $\lambda_{0}+2 \leq k_{+}<9 / 2$,

$$
\begin{gathered}
t^{-1}\left\|\partial_{t} W\right\|_{6}\left\|B_{S}(W, W)\right\|_{3} \leq C t^{-1}\left\|\nabla \partial_{t} W\right\|_{2}\left\|\omega^{1 / 2} B_{S}(W, W)\right\|_{2} \\
\leq C t^{-3 / 2}\left(t^{\beta\left(k_{+}+1 / 2\right)}+t^{(\beta+3) / 2} 1_{0}\right) \leq C t^{\lambda_{0}-2}
\end{gathered}
$$

for $\lambda_{0} \geq \beta\left(k_{+}+1\right)$, by (3.76) and (3.82) with $m=1 / 2$,

$$
\begin{gathered}
t^{-1}\left\|\widetilde{B}_{S}\left(\partial_{t} w_{0}, w_{0}\right)\right\|_{2} \leq C t^{-1+\beta\left(k_{+}-1\right)} I_{k_{+}-1}\left(\left\|\omega^{k_{+}} w_{0}\right\|_{2}\left\|w_{0}\right\|_{\infty}\right. \\
\left.+\left\|\Delta w_{0}\right\|_{2}\left\|\omega^{k_{+}-2} w_{0}\right\|_{\infty}\right) \leq C t^{-1+\beta\left(k_{+}-1\right)} \leq C t^{\lambda_{0}-2}
\end{gathered}
$$

for $k_{+} \geq 2$ and $\lambda_{0} \leq \beta\left(k_{+}-1\right)+1$. The last condition for $k_{+} \geq 2$ follows from $\lambda_{0} \leq 3 / 2$ if $\beta \geq 1 / 2$ and from $\lambda_{0} \leq \beta\left(k_{+}+1\right)$ if $\beta \leq 1 / 2$.

$$
\begin{gathered}
t^{-1}\left\|\widetilde{B}_{S}\left(\partial_{t} w_{0}, w_{1}+w_{2}\right)\right\|_{2} \leq C t^{-1+\beta / 2} I_{1 / 2}\left(\left\|\partial_{t} w_{0}\right\|_{6}\left\|w_{1}+w_{2}\right\|_{2}\right) \\
\leq C t^{\beta / 2}\left(1-\ell n t+t^{1 / 2} 1_{0}\right) \leq C t^{\lambda_{0}-2}
\end{gathered}
$$

where the last inequality is largely satisfied for $\lambda_{0} \leq 3 / 2$. From (3.85)-(3.90) and the fact that the other terms in (3.85) are correctly estimated by the previous remark, it follows that $R_{1}$ satisfies the estimate (3.24) for $j=1$.

The estimate (3.25) of $R_{2}$ is the same as in II and we briefly recall its proof for completeness. From (3.59) it follows that $R_{20}$ satisfies (3.25) provided $\lambda_{0}<2+\beta$. We next consider $R_{2 \nu}$ defined by (3.51). For $m \geq 0$, we estimate

$$
t^{-1}\left\|\omega^{m+1} B_{0 L}\right\|_{2} \leq C t^{1+\mu-\beta(m+5 / 2+\mu)} \leq C t^{\lambda_{0}-1-\beta m}
$$

by (3.62) provided

$$
\beta(5 / 2+\mu) \leq 2+\mu-\lambda_{0}
$$

which is half of (3.68), and

$$
\begin{aligned}
t^{-1} \| \omega^{m+1} B_{L}(W & \left.+W_{1}, w_{2}\right) \|_{2} \leq t^{-1-\beta m} I_{0}\left(\left\|W+W_{1}\right\|_{\infty}\left\|w_{2}\right\|_{2}\right) \\
& \leq C t^{1 / 2-\beta m} 1_{0} \leq C t^{\lambda_{0}-1-\beta m}
\end{aligned}
$$

under the condition (3.84) which is the other half of (3.68). This completes the proof of the estimates (3.20)-(3.25) and therefore of Proposition 3.2. 
Remark 3.2. The parameters $\beta, \ell \lambda_{0}, k_{+}$and $\mu$ play the same role and basically satisfy the same conditions as in II. The parameter $\beta$ fixes the splitting of $B$ into long and short range parts and thereby fixes the auxiliary system (1.20). The parameters $\ell$ and $\lambda_{0}$ fix the function space where that system is solved. The regularity of $w$ is already fixed, $\ell$ fixes the regularity of $s$ and $\lambda_{0}$ fixes the rates of convergence in time. The parameters $k_{+}$and $\mu$ fix the regularity of $w_{+}$and the vanishing of $\left(F A_{+}, F \dot{A}_{+}\right)$at $\xi=0$, which have to be sufficient, as expressed by the lower bounds (3.67) (3.68). The condition (3.68) is the combination of (3.84) (3.92), which coincide with (II.3.81) and (II.3.82) respectively, with equality allowed and with $\beta_{0}=\beta$.

Remark 3.3. The conditions on the parameters become simpler in the special case $\beta=1 / 3$ which optimizes (3.68). If one takes in addition $3 / 2<\ell \leq 2$, the remaining conditions reduce to

$$
1<\lambda_{0}<3 / 2 \quad, \quad k_{+} \geq \lambda_{0}+2 \quad, \quad \mu \geq(3 / 2) \lambda_{0}-7 / 4(>-1 / 4) .
$$

Remark 3.4. The condition $k_{+} \geq \lambda_{0}+2$ is used only to estimate the term $\nabla h \cdot \partial_{t} \nabla w_{0}$ in the estimate of $\partial_{t} R_{1}$ in $L^{2}$ (see (3.87)). Everywhere else the condition $k_{+} \geq 3$ is sufficient. The latter condition would also be sufficient for that term if one were using a better estimate of $\|\nabla h\|_{\infty}$ than follows from (3.63) and Sobolev inequalities (see Proposition 7.4 in [4] for estimates of this type).

We finally comment briefly on the condition $(3.61)_{\mu}$ which restricts the behaviour of $\left(F A_{+}, F \dot{A}_{+}\right)$at $\xi=0$. That condition can be ensured by assuming sufficient decay of $\left(A_{+}, \dot{A}_{+}\right)$at infinity in space, possibly supplemented by some moment conditions. We refer for details to the discussion at the end of Section II.3, from which we extract the following minor variation of Lemma II.3.5, which is typical of the situation.

Lemma 3.5. Let $-1 / 2 \leq \mu<1$. Let $\left(A_{+}, \dot{A}_{+}\right)$satisfy (3.60) and in addition

$$
\begin{gathered}
x^{2} A_{+} \in L^{2 \vee 3 /(\mu+1)} \quad, \quad x^{2} \dot{A}_{+}, x A_{+} \in L^{3 /(2+\mu)} \\
<x>^{1+\mu+\varepsilon} \dot{A}_{+} \in L^{1} \quad, \int \dot{A}_{+} d x=0, \\
A_{+}, x \dot{A}_{+} \in L^{3 /(3+\mu)} \quad \text { for } \mu<0, \\
\int A_{+} d x=\int x \dot{A}_{+} d x=0 \quad, \quad<x>^{\mu+\varepsilon} A_{+} \in L^{1} \quad \text { for } \mu \geq 0,
\end{gathered}
$$

for some $\varepsilon>0$. Then $(3.61)_{\mu}$ holds.

\section{Wave operators and asymptotics for $(\mathrm{u}, \mathrm{A})$}

In this section we complete the construction of the wave operators for the system (1.1) (1.2) and we derive asymptotic properties of solutions in their range. The construction relies in an essential way on Proposition 3.2. So far we have worked with the system $(1.20)$ for $(w, s)$ and the first task is to reconstruct the phase $\varphi$. Corresponding to $S=s_{0}+s_{1}$, we define $\phi=\varphi_{0}+\varphi_{1}$ where

$$
\varphi_{0}=\int_{t}^{1} d t^{\prime} t^{\prime-1} B_{L}\left(w_{0}\left(t^{\prime}\right), w_{0}\left(t^{\prime}\right)\right)
$$




$$
\varphi_{1}=\int_{0}^{t} d t^{\prime}(1 / 2)\left|s_{0}\left(t^{\prime}\right)\right|^{2}-2 \int_{0}^{t} d t^{\prime} t^{\prime-1} B_{L}\left(w_{0}\left(t^{\prime}\right), w_{1}\left(t^{\prime}\right)\right),
$$

so that $s_{0}=\nabla \varphi_{0}$ and $s_{1}=\nabla \varphi_{1}$.

Let now $(w, s)$ be the solution of the system (1.20) constructed in Proposition 3.1 and let $(q, \sigma)=(w, s)-(W, S)$. We define*

$$
\psi=\int_{0}^{t} d t^{\prime}(1 / 2)\left(\sigma \cdot(\sigma+2 S)+s_{1} \cdot\left(s_{1}+2 s_{0}\right)\right)\left(t^{\prime}\right)
$$

$$
-\int_{0}^{t} d t^{\prime} t^{\prime-1}\left(B_{0 L}+B_{L}(q, q)+2 B_{L}(W, q)+B_{L}\left(w_{1}, w_{1}\right)+B_{L}\left(W+W_{1}, w_{2}\right)\right)\left(t^{\prime}\right)
$$

which is taylored to ensure that $\nabla \psi=\sigma$, given the fact that $s_{0}, s_{1}$ and $\sigma$ are gradients. The integral is easily seen to converge in $\dot{H}^{1}$ (see (I.8.4) and (3.91)(3.93) with $m=0$ ), and to satisfy

$$
\|\nabla \psi\|_{2}=\|\sigma\|_{2} \leq C t^{\lambda_{0}} .
$$

Finally we define $\varphi=\phi+\psi$ so that $\nabla \varphi=s$, and $(w, \varphi)$ solves the system (1.18). For more details on the reconstruction of $\varphi$ from $s$, we refer to Section 8 of I.

We can now define the wave operators for the system (1.1) (1.2) as follows. We start from the asymptotic state $\left(u_{+}, A_{+}, \dot{A}_{+}\right)$for $(u, A)$. We define $w_{+}=F u_{+}$, we define $B_{0}$ by (1.4) (1.13), namely

$$
A_{0}=\dot{K}(t) A_{+}+K(t) \dot{A}_{+}=t^{-1} D_{0}(t) B_{0}(1 / t),
$$

and we define $(W, S)$ by $(3.42)-(3.47)$.

We next solve the system (1.20) with initial time zero by Proposition 3.2 and we reconstruct $\varphi$ from $s$ as explained above, namely $\varphi=\varphi_{0}+\varphi_{1}+\psi$ with $\varphi_{0}, \varphi_{1}$ and $\psi$ defined by (4.1) (4.2) (4.3) with $(q, \sigma)=(w, s)-(W, S)$. We finally substitute $(w, \varphi)$ thereby obtained into (1.11) (1.3) thereby obtaining a solution $(u, A)$ of the system (1.1) (1.2). The wave operator is defined as the map $\Omega:\left(u_{+}, A_{+}, \dot{A}_{+}\right) \rightarrow(u, A)$.

We now turn to the study of the asymptotic properties of $(u, A)$ and in particular of its convergence to its asymptotic form $\left(u_{a}, A_{a}\right)$ defined in a natural way (compare with (1.3) (1.11)) by

$$
\begin{gathered}
u_{a}(t)=M(t) D(t) \exp (i \phi(1 / t)) \bar{W}(1 / t) \\
A_{a}(t)=A_{0}(t)+A_{1}\left(|D(t) W(1 / t)|^{2}\right) .
\end{gathered}
$$

In order to compare $u$ with $u_{a}$, we need some estimates of the difference $\exp (-i \varphi) w-$ $\exp (-i \phi) W$. The following lemma is based on part of the estimates of Proposition 3.2 but does not assume that $(w, \nabla \varphi)$ is a solution of the auxiliary system (1.20).

Lemma 4.1. Let $0<\beta<1, \lambda_{0}>1,0<\tau \leq 1$ and $I=(0, \tau]$. Let $W \in$ $\mathcal{C}\left(I, H^{2}\right) \cap \mathcal{C}^{1}\left(I, H^{1}\right)$ satisfy (3.20) (3.21) and

$$
\|\Delta W\|_{2} \leq a_{1} t^{-1 / 2}
$$

for all $t \in I$. Let $(w, \nabla \psi) \in \mathcal{C}\left(I, H^{2} \oplus H^{1}\right) \cap \mathcal{C}^{1}\left(I, L^{2} \oplus L^{2}\right)$ (with $\left.\psi, \partial_{t} \psi \in \mathcal{C}\left(I, L^{6}\right)\right)$ satisfy the estimates

$$
\|w(t)-W(t)\|_{2} \leq C t^{\lambda_{0}}
$$

${ }^{*}$ We take this opportunity to correct an omission in II, where the terms $B_{0 L}$ and $B_{L}(W+$ $\left.W_{1}, w_{2}\right)$ are missing in (II.4.3). This has no incidence on the rest of II. 


$$
\left\|\partial_{t}(w(t)-W(t))\right\|_{2} \vee\|\Delta(w(t)-W(t))\|_{2} \leq C t^{\lambda_{0}-1}
$$

$$
\left\|\nabla^{m+1} \partial_{t}^{j} \psi(t)\right\|_{2} \leq C t^{\lambda_{0}-j-\beta m} \quad \text { for } j, m=0,1, j+m \leq 1
$$

for all $t \in I$.

(1) Let $f=\exp (-i \psi) w-W$. Then the following estimates hold for all $t \in I$ :

$$
\|f(t)\|_{2} \leq C t^{\lambda_{0}}
$$

$$
\left\|\partial_{t} f(t)\right\|_{2} \vee\|\Delta f(t)\|_{2} \leq C t^{\lambda_{0}-1} .
$$

(2) Let $\phi \in \mathcal{C}\left(I, W_{\infty}^{2}\right) \cap \mathcal{C}^{1}\left(I, L^{\infty}\right)$ satisfy the estimates

$$
\left\{\begin{array}{l}
\left\|\partial_{t} \phi\right\|_{\infty} \leq C t^{-1} \\
\|\nabla \phi\|_{\infty} \vee\|\Delta \phi\|_{\infty} \leq C(1-\ln t)
\end{array}\right.
$$

for all $t \in I$. Let $\varphi=\phi+\psi$ and $g=\exp (-i \phi) f=\exp (-i \varphi) w-\exp (-i \phi) W$. Then the following estimates hold for all $t \in I$ :

$$
\begin{gathered}
\|g(t)\|_{2} \leq C t^{\lambda_{0}} \\
\left\|\partial_{t} g(t)\right\|_{2} \vee\|\Delta g(t)\|_{2} \leq C t^{\lambda_{0}-1} .
\end{gathered}
$$

Proof. Part (1). We write

$$
f=(\exp (-i \psi)-1) w+w-W
$$

and we estimate

$$
\begin{gathered}
\|f\|_{2} \leq\|\psi\|_{6}\|w\|_{3}+\|w-W\|_{2} \\
\leq C\|\nabla \psi\|_{2}\|w\|_{3}+\|w-W\|_{2} \leq C t^{\lambda_{0}} .
\end{gathered}
$$

Next

$$
\partial_{t} f=-i \exp (-i \psi)\left(\partial_{t} \psi\right) w+\exp (-i \psi) \partial_{t}(w-W)+(\exp (-i \psi)-1) \partial_{t} W
$$

so that

$\left\|\partial_{t} f\right\|_{2} \leq\left\|\partial_{t} \psi\right\|_{6}\|w\|_{3}+\left\|\partial_{t}(w-W)\right\|_{2}+\|\psi\|_{6}\left\|\partial_{t} W\right\|_{3}$

$$
\leq C\left(t^{\lambda_{0}-1}+t^{\lambda_{0}-1 / 2}\right) \leq C t^{\lambda_{0}-1}
$$

by (3.20) (3.21) and (4.8)-(4.10).

Finally

so that

$$
\begin{aligned}
\Delta f= & -\exp (-i \psi)\left(i \Delta \psi+|\nabla \psi|^{2}\right) w-2 i \exp (-i \psi) \nabla \psi \cdot \nabla w \\
& +\exp (-i \psi) \Delta(w-W)+(\exp (-i \psi)-1) \Delta W
\end{aligned}
$$

$$
\begin{gathered}
\|\Delta f\|_{2} \leq\left(\|\Delta \psi\|_{2}+\|\nabla \psi\|_{4}^{2}\right)\|w\|_{\infty}+2\|\nabla \psi\|_{6}\|\nabla w\|_{3} \\
+\|\Delta(w-W)\|_{2}+\|\psi\|_{\infty}\|\Delta W\|_{2} \\
\leq C\left(t^{\lambda_{0}-\beta}+t^{2 \lambda_{0}-3 \beta / 2}+t^{\lambda_{0}-1}+t^{\lambda_{0}-(\beta+1) / 2}\right) \leq C t^{\lambda_{0}-1}
\end{gathered}
$$

by (3.20) and (4.7)-(4.10).

Part 2. (4.14) is obvious. Next

$$
\begin{gathered}
\left\|\partial_{t} g\right\|_{2} \leq\left\|\partial_{t} \phi\right\|_{\infty}\|f\|_{2}+\left\|\partial_{t} f\right\|_{2} \leq C t^{\lambda_{0}-1} \\
\|\Delta g\|_{2} \leq\left(\|\Delta \phi\|_{\infty}+\|\nabla \phi\|_{\infty}^{2}\right)\|f\|_{2}+2\|\nabla \phi\|_{\infty}\|\nabla f\|_{2}+\|\Delta f\|_{2}
\end{gathered}
$$




$$
\leq C\left(t^{\lambda_{0}}(1-\ell n t)^{2}+t^{\lambda_{0}-1 / 2}(1-\ell n t)+t^{\lambda_{0}-1}\right) \leq C t^{\lambda_{0}-1} .
$$

In order to state the asymptotic properties of $u$, it is convenient to use the related function (compare with (1.7) (1.11))

$$
\widetilde{u}(t)=U(-t) u(t)=M(t)^{*} F^{*} \exp (i \varphi(1 / t)) \bar{w}(1 / t)
$$

and its asymptotic form

$$
\widetilde{u}_{a}(t)=U(-t) u_{a}(t)=M(t)^{*} F^{*} \exp (i \phi(1 / t)) \bar{W}(1 / t) .
$$

We can now state the asymptotic properties of $(u, A)$.

Proposition 4.1. Let $0<\beta<3 / 5, \ell>3 / 2$ and $1 \vee \beta(\ell+1)<\lambda_{0}<3 / 2$. Let $k_{+}$and $\mu$ satisfy (3.67) (3.68), let $w_{+}=F u_{+} \in H^{k_{+}}$, let $\left(A_{+}, \dot{A}_{+}\right)$satisfy (2.16) (3.60) (3.61) $)_{\mu}$. Let $(W, S)$ be defined by (3.42) (3.47). Let $(w, s)$ be the solution of the system (1.20) in $(0, \tau]$ obtained in Proposition 3.2 and let $I=[T, \infty)$ where $T=\tau^{-1}$. Let $\phi=\varphi_{0}+\varphi_{1}$ and $\varphi=\phi+\psi$ be defined by (4.1)-(4.3). Define (u,A) by (1.3)-(1.5) (1.11), define $\left(u_{a}, A_{a}\right)$ by (4.5) (4.6) and $\left(\widetilde{u}, \widetilde{u}_{a}\right)$ by (4.16) (4.17). Then $\widetilde{u} \in \mathcal{C}\left(I, F H^{2}\right) \cap \mathcal{C}^{1}\left(I, L^{2}\right), A \in \mathcal{C}\left(I, H^{1}\right) \cap \mathcal{C}^{1}\left(I, L^{2}\right),(u, A)$ solves the system (1.1) (1.2) in $I$ and $(u, A)$ behaves asymptotically in time as $\left(u_{a}, A_{a}\right)$ in the sense that the following estimates hold for all $t \in I$ :

$$
\begin{gathered}
\left\|\partial_{t}^{j}\left(\widetilde{u}(t)-\widetilde{u}_{a}(t)\right)\right\|_{2} \leq C\left(a_{+}, A_{+}, \dot{A}_{+}\right) t^{-\lambda_{0}-j} \quad \text { for } j=0,1, \\
\left\|x^{2}\left(\widetilde{u}(t)-\widetilde{u}_{a}(t)\right)\right\|_{2} \leq C\left(a_{+}, A_{+}, \dot{A}_{+}\right) t^{-\lambda_{0}+1}, \\
\left\|u(t)-u_{a}(t)\right\|_{r} \leq C\left(a_{+}, A_{+}, \dot{A}_{+}\right) t^{-\lambda_{0}+\delta(r) / 2} \quad \text { for } 2 \leq r \leq \infty .
\end{gathered}
$$

Furthermore $A-A_{a} \in \mathcal{C}\left(I, H^{3}\right)$ and the following estimates hold for all $t \in I$ :

$$
\begin{gathered}
\left\|A(t)-A_{a}(t)\right\|_{2} \leq C\left(a_{+}, A_{+}, \dot{A}_{+}\right) t^{-\lambda_{0}+1 / 2} \\
\left\|\omega^{m+1}\left(A(t)-A_{a}(t)\right)\right\|_{2} \leq C\left(a_{+}, A_{+}, \dot{A}_{+}\right) t^{-\lambda_{0}-1 / 2-m / 2}\left(1+t^{m / 2-3 / 4}\right)
\end{gathered}
$$

for $0 \leq m \leq 2$. Here $a_{+}=\left|w_{+}\right|_{k_{+}}$and the constants $C\left(a_{+}, A_{+}, \dot{A}_{+}\right)$depend on $\left(A_{+}, \dot{A}_{+}\right)$through the norms associated with (2.16) (3.60) (3.61) $)_{\mu}$.

Proof. The regularity of $u$ follows immediately from that of $(w, \varphi)$ implied by Proposition 3.2, from the definition of $\varphi$, from (4.16) and from the commutation relations

$$
\left\{\begin{array}{l}
x^{2} M^{*} F^{*}=-M^{*} F^{*} \Delta \\
i \partial_{t} M^{*} F^{*}=M^{*} F^{*}\left(i \partial_{t}+\left(2 t^{2}\right)^{-1} \Delta\right) .
\end{array}\right.
$$

The estimates (4.18) (4.19) follow from Lemma 4.1, more precisely from (4.14) (4.15) and from (4.23) again, once we have verified the assumptions of that lemma. Now (4.7) follows from Lemma 3.3, especially (3.52) (3.55) and from (3.74) with $m=2$. The estimates (4.8) (4.9) are a rewriting of (3.69) (3.70), while (4.10) is a special case of (3.71) (3.72) with $m=0,1$. Finally (4.13) follows from the definition of $\phi$ and from Lemma 3.3, which implies that

$$
\left\|\omega^{m+1} \phi\right\|_{2} \leq C(1-\ell n t)
$$


for $0 \leq m \leq 3 \leq k_{+}$.

$$
\left\|\omega^{m+1} \partial_{t} \phi\right\|_{2} \leq C t^{-1}
$$

The estimate (4.20) follows from the fact that

$$
u(t)-u_{a}(t)=M(t) D(t) \bar{g}(1 / t)
$$

where $g$ is defined in Lemma 4.1, so that for $2 \leq r \leq \infty$

$$
\begin{gathered}
\left\|u(t)-u_{a}(t)\right\|_{r}=t^{-\delta(r)}\|g(1 / t)\|_{r} \\
\leq C t^{-\delta(r)}\|g(1 / t)\|_{2}^{1-\delta(r) / 2}\|\Delta g(1 / t)\|_{2}^{\delta(r) / 2} \leq C t^{-\lambda_{0}-\delta(r) / 2}
\end{gathered}
$$

by (4.14) (4.15).

The regularity properties of $A$ follow immediately from the assumptions on $\left(A_{+}, \dot{A}_{+}\right)$, from (1.13)-(1.15) and from the regularity of $w$ implied by Proposition 3.2. In order to derive the estimates (4.21) (4.22), we note that

$$
A(t)-A_{a}(t)=t^{-1} D_{0}(t) B_{1}(q, q+2 W)(1 / t)
$$

with $q=w-W$. We estimate

$$
\left\|B_{1}(q, q+2 W)(t)\right\|_{2} \leq C I_{-1}\left(\|q\|_{2}\|q+2 W\|_{3}\right) \leq C t^{\lambda_{0}}
$$

by (2.8) and (3.69),

$$
\begin{gathered}
\left\|\omega^{m+1} B_{1}(q, q+2 W)(t)\right\|_{2} \leq C I_{m}\left(\left\|\omega^{m} q\right\|_{2}\|q+2 W\|_{\infty}\right. \\
\left.+\left\|\omega^{\delta} q\right\|_{2}\left\|\omega^{m} w_{0}\right\|_{3 / \delta}+\|q\|_{\infty}\left(\left\|\omega^{m} w_{1}\right\|_{2}+\left\|\omega^{m} w_{2}\right\|_{2}\right)\right)
\end{gathered}
$$

by $(2.8)$ and Lemma 2.2 , with $0<\delta<3 / 2$,

$$
\cdots \leq C\left(t^{\lambda_{0}-m / 2}+t^{\lambda_{0}-\delta / 2}+t^{\lambda_{0}-3 / 4}\left(t(1-\ell n t)+t^{3 / 2-m} 1_{m}\right)\right)
$$

for $0 \leq m \leq 2$ and $\delta \geq m-3 / 2$, by (3.69) (3.70) (3.52) (3.55) (3.74),

$$
\cdots \leq C t^{\lambda_{0}-m / 2}\left(1+t^{3 / 4-m / 2}\right) \text {. }
$$

Combining (4.28) (4.29) with $t$ changed into $1 / t$ and (4.27) yields (4.21) (4.22).

We conclude this section with some comments on the relation between this paper and I, II. In all cases the regularity of $w$ includes $w \in \mathcal{C}\left(H^{k}\right)$, with $1<k<2$ and a support condition on $F u_{+}$in $I$, and with $1<k<3 / 2$ and no support condition in II. The present paper covers the case $k=2$ with no support condition. In II, the correction term $w_{2}$ allowing to eliminate the support condition could be omitted in the statement of the final result (see Proposition II.4.1, part (3)) because $w_{2}$ satisfies the same estimates as $q$ (namely (II.4.8) (II.4.9) with $q=w-W$ ). This is no longer the case here, and $w_{2}$ has to be kept in the final result. The reason is that whereas $\left\|w_{2}\right\|_{2}$ and $\left\|\partial_{t} w_{2}\right\|_{2}$ are estimated as $\|q\|_{2}$ and $\left\|\partial_{t} q\right\|_{2}$, we have only $\left\|\Delta w_{2}\right\|_{2} \leq C t^{-1 / 2}$ by (3.74), as compared with $\|\Delta q\|_{2} \leq C t^{\lambda_{0}-1}$.

We finally mention two minor differences in the statement of the asymptotic properties of $u$. In I, II we have used the operator $J=x+i t \nabla$ and norms of the type $\left\||J(t)|^{k} u\right\|_{2}$. By the commutation relation

$$
J(t)=U(t) x U(-t)
$$

this is equivalent to $\left\||x|^{k} \widetilde{u}\right\|_{2}$. We have used $\widetilde{u}$ in the present paper because it allows for simpler statements when considering time derivatives. The second minor 
difference is that in I, II we have stated the convergence of $u$ to $u_{a}$ in terms of $\exp (i \phi)\left(u-u_{a}\right)$ instead of $u-u_{a}$. We could as well have stated them also in terms of $u-u_{a}$ by using a suitable variant of Lemma 4.1, part (3). This possibility arises mainly because the WS system belongs to the borderline long range case, where the correcting phase $\phi$ is only logarithmic.

\section{References}

[1] J. Ginibre, G. Velo : Long range scattering and modified wave operators for the WaveSchrödinger system, Ann. H.P., 3 (2002), 537-612.

[2] J. Ginibre, G. Velo : Long range scattering and modified wave operators for the WaveSchrödinger system II, Ann. H.P., 4 (2003), 973-999.

[3] J. Ginibre, G. Velo : Long range scattering and modified wave operators for the MaxwellSchrödinger system I. The case of vanishing asymptotic magnetic field, Commun. Math. Phys., 236 (2003), 395-448.

[4] J. Ginibre, G. Velo : Scattering theory for the Schrödinger equation in some external time dependent magnetic fields, preprint, math.AP/0401355, J. Diff. Eq., in press.

[5] J. Ginibre, G. Velo : Long range scattering for the Wave-Schrödinger system with large wave data and small Schrödinger data, preprint, math. AP/0406608, Hokkaido Math. J., in press.

[6] J. Ginibre, G. Velo : Long range scattering for the Maxwell-Schrödinger system with large magnetic field data and small Schrödinger data, preprint, math. AP/0407017.

[7] J. Ginibre, G. Velo : Long range scattering for some Schrödinger related nonlinear systems, preprint, math.AP/0412430.

[8] L. Hörmander : The Analysis of Linear Partial Differential Operators, Vol I, Springer, Berlin, 1983.

[9] T. Kato, G. Ponce : Commutator estimates and the Euler and Navier-Stokes equations, Comm. Pure Appl. Math., 41 (1988), 891-907.

[10] C. Kenig, G. Ponce, L. Vega : The initial value problem for a class of nonlinear dispersive equations, in Functional-Analytic Methods for Partial Differential Equations, Lect. Notes Math., 1450 (1990), 141-156.

[11] T. Ozawa : Long range scattering for nonlinear Schrödinger equations in one space dimension, Commun. Math. Phys., 139 (1991), 479-493.

[12] T. Ozawa, Y. Tsutsumi : Asymptotic behaviour of solutions for the coupled Klein-GordonSchrödinger equations, in Spectral and Scattering Theory and Applications, Adv. Stud. in Pure Math., Jap. Math. Soc., 23 (1994), 295-305.

[13] A. Shimomura : Wave operators for the coupled Klein-Gordon-Schrödinger equations in two space dimensions, Funkcial. Ekvac., 47 (2004), 63-82.

[14] A. Shimomura : Modified wave operators for the coupled Wave-Schrödinger equations in three space dimensions, Disc. Cont. Dyn. Syst., 9 (2003), 1571-1586.

[15] Y. Tsutsumi : Global existence and asymptotic behaviour of solutions for the MaxwellSchrödinger system in three space dimensions, Commun. Math. Phys., 151 (1993), 543-576.

Laboratoire de Physique Théorique, Université de Paris Xi, BÂtiment 210, F-91405 Orsay Cedex, France

E-mail address: jean.ginibre@th.u-psud.fr

Dipartimento di Fisica, Università di Bologna, and INFn, Sezione di Bologna, Italy E-mail address: velo@bo.infn.it 